\title{
Welcome to the Twilight Zone: The Mid-infrared Properties of Post-starburst Galaxies
}

\author{
Katherine Alatalo ${ }^{1,16}$, Theodoros Bitsakis ${ }^{2}$, Lauranne Lanz ${ }^{3,4}$, Mark Lacy ${ }^{5}$, Michael J. I. Brown ${ }^{6,7,17}$, K. Decker French ${ }^{8}$, \\ Laure Ciesla $^{9}$, Philip N. Appleton ${ }^{5}$, Rachael L. Beaton ${ }^{1}$, Sabrina L. Cales ${ }^{10}$, Jacob Crossett ${ }^{6}$, Jesús Falcón-Barroso ${ }^{11,12}$, \\ Daniel D. Kelson ${ }^{1}$, Lisa J. Kewley ${ }^{13}$, Mariska Kriek ${ }^{14}$, Anne M. Medling ${ }^{13,15,16}$, John S. Mulchaey ${ }^{1}$, Kristina Nyland ${ }^{5}$, \\ Jeffrey A. Rich ${ }^{1}$, and C. Meg Urry ${ }^{10}$ \\ ${ }^{1}$ The Observatories of the Carnegie Institution for Science, 813 Santa Barbara Street, Pasadena, CA 91101, USA; kalatalo@carnegiescience.edu \\ ${ }^{2}$ Instituto de Radioastronomía y Astrofísica, Universidad Nacional Autónoma de México, C.P. 58190, Morelia, Mexico \\ ${ }^{3}$ Infrared Processing and Analysis Center, California Institute of Technology, Pasadena, California 91125, USA \\ ${ }_{4}$ Dartmouth College, 6127 Wilder Laboratory, Hanover, NH 03755, USA \\ ${ }^{5}$ National Radio Astronomy Observatory, 520 Edgemont Road, Charlottesville, VA 22903, USA \\ ${ }^{6}$ School of Physics, Monash University, Clayton, Victoria 3800, Australia \\ ${ }^{7}$ Monash Centre for Astrophysics, Monash University, Clayton, Victoria 3800, Australia \\ ${ }^{8}$ Steward Observatory, University of Arizona, 933 North Cherry Avenue, Tucson, AZ 85721, USA \\ ${ }^{9}$ Laboratoire AIM-Paris-Saclay, CEA/DSM/Irfu-CNRS-Université Paris Diderot, CEA-Saclay, F-91191 Gif-sur-Yvette, France \\ ${ }^{10}$ Yale Center for Astronomy and Astrophysics, Physics Department, Yale University, New Haven, CT 06511, USA \\ ${ }^{11}$ Instituto de Astrofísica de Canarias, E-38205 La Laguna, Tenerife, Spain \\ ${ }^{12}$ Departamento de Astrofísica, Universidad de La Laguna (ULL), E-38200 La Laguna, Tenerife, Spain \\ ${ }^{13}$ Research School of Astronomy and Astrophysics, Australian National University, Cotter Road, Weston ACT 2611, Australia \\ ${ }^{14}$ Department of Astronomy, Campbell Hall, University of California, Berkeley, CA 94720, USA \\ ${ }^{15}$ California Institute of Technology, MC 249-17, 1200 East California Boulevard, Pasadena, CA 91125, USA \\ Received 2016 July 30; revised 2017 May 8; accepted 2017 May 11; published 2017 June 26
}

\begin{abstract}
We investigate the optical and Wide-field Survey Explorer (WISE) colors of "E+A" identified post-starburst galaxies, including a deep analysis of 190 post-starbursts detected in the $2 \mu \mathrm{m}$ All Sky Survey Extended Source Catalog. The post-starburst galaxies appear in both the optical green valley and the WISE Infrared Transition Zone. Furthermore, we find that post-starbursts occupy a distinct region of [3.4]-[4.6] versus [4.6]-[12] WISE colors, enabling the identification of this class of transitioning galaxies through the use of broadband photometric criteria alone. We have investigated possible causes for the WISE colors of post-starbursts by constructing a composite spectral energy distribution (SED), finding that the mid-infrared $(4-12 \mu \mathrm{m})$ properties of post-starbursts are consistent with either $11.3 \mu \mathrm{m}$ polycyclic aromatic hydrocarbon emission, or thermally pulsating asymptotic giant branch (TP-AGB) and post-AGB stars. The composite SED of extended post-starburst galaxies with $22 \mu \mathrm{m}$ emission detected with signal-to-noise ratio $\geqslant 3$ requires a hot dust component to produce their observed rising midinfrared SED between 12 and $22 \mu \mathrm{m}$. The composite SED of WISE $22 \mu \mathrm{m}$ non-detections $(\mathrm{S} / \mathrm{N}<3)$, created by stacking $22 \mu \mathrm{m}$ images, is also flat, requiring a hot dust component. The most likely source of the mid-infrared emission of these $\mathrm{E}+\mathrm{A}$ galaxies is a buried active galactic nucleus (AGN). The inferred upper limits to the Eddington ratios of post-starbursts are $10^{-2}-10^{-4}$, with an average of $10^{-3}$. This suggests that AGNs are not radiatively dominant in these systems. This could mean that including selections capable of identifying AGNs as part of a search for transitioning and post-starburst galaxies would create a more complete census of the transition pathways taken as a galaxy quenches its star formation.
\end{abstract}

Key words: galaxies: evolution - galaxies: star formation - galaxies: stellar content - infrared: galaxies

Supporting material: extended figure

\section{Introduction}

Galaxies in the modern universe show two bimodal distributions, one in morphology and one in color space. Hubble (1926) discussed a "tuning fork" to classify the morphologies of galaxies, representing spiral (late-type) galaxies and elliptical and lenticular (early-type) galaxies. In color space, galaxies break into a blue cloud and a red sequence (Baade 1958; Holmberg 1958; Tinsley 1978; Strateva et al. 2001; Baldry et al. 2004), with a genuine dearth of galaxies with intermediate colors in the so-called "green valley." This dearth is used to suggest that galaxies undergoing the metamorphosis between blue spirals and red early-types must be rapid.

\footnotetext{
${ }^{16}$ Hubble fellow.

17 Future ARC fellow.
}

Once $z \approx 0$ galaxies begin the process of transitioning, the probability that it is a one-way process is high (Appleton et al. 2013; Young et al. 2014), with very few circumstances under which the galaxy will transition back permanently (Kannappan et al. 2013). Because of this, it is essential to understand all possible pathways and physical mechanisms that can trigger a galaxy's metamorphosis. Many pathways to transformation have been observed, though it is likely that the list is not exhaustive.

Mergers are capable of driving the molecular gas into the center, allowing it to be consumed in a starburst, and heating the stellar disks of the interacting galaxies (Toomre \& Toomre 1972; Springel et al. 2005), creating an elliptical galaxy. Minor mergers also appear capable of quenching star formation $(\mathrm{Qu}$ et al. 2010; Eliche-Moral et al. 2012; Alatalo et al. 2014b), especially if the recipient galaxy endures many minor mergers over its lifetime. Secular evolution, in which a galaxy bulge 
grows sufficiently large to stabilize a molecular disk against gravitational collapse (thus inhibiting star formation), has also been shown in simulations to quench galaxies (Martig et al. 2009), with additional observational evidence manifesting in early-type galaxies (Martig et al. 2013; Davis et al. 2014).

When galaxies fall into a cluster potential, they suffer strangulation, in which their ability to accrete external gas and replenish their supply is stunted (Bekki et al. 2002; Blanton \& Moustakas 2009), truncating star formation. They can also suffer harassment, in which gravitational torques from other cluster members dynamically heat the stars (Mihos 1995; Moore et al. 1996; Bekki 1998). Group interactions (Hickson et al. 1992; Zabludoff \& Mulchaey 1998) are able to catalyze quenching, which has been observed through the study of the individual group galaxies (Johnson et al. 2007; Bitsakis et al. 2011, 2014, 2016; Martinez-Badenes et al. 2012; Lisenfeld et al. 2014; Alatalo et al. 2015a) and the evolution of the intragroup medium (Verdes-Montenegro et al. 2001; Rasmussen et al. 2008; Borthakur et al. 2010). Additionally, it is possible that much of the galaxy transformation observed in the cluster environment takes place during a group pre-processing phase (Dressler et al. 2013).

Active galactic nucleus (AGN) feedback, introduced to explain the truncated mass function of galaxies (Silk \& Rees 1998; Di Matteo. et al. 2005; Croton et al. 2006; Oppenheimer et al. 2010), can rapidly expel star-forming fuel from the galaxy and quickly quench star formation (Hopkins et al. 2006, 2008). Molecular gas outflows detected in some AGN hosts may be a signature of AGN feedback (Feruglio et al. 2010; Fischer et al. 2010; Alatalo et al. 2011; Sturm et al. 2011; Aalto et al. 2012, 2016; Cicone et al. 2012, 2014), though the nearby examples do not appear to be powerful enough to rapidly eject the interstellar medium from the host, instead mainly injecting turbulence into the existing gas (Alatalo et al. 2015b; Guillard et al. 2015; Lanz et al. 2015, 2016; Costagliola et al. 2016) and ultimately depleting molecular gas at a rate consistent with the star formation rate (Alatalo 2015). It is possible that radiationmode AGN feedback provides sufficient energy to quench a galaxy at high redshift (Zakamska et al. 2016), but the mechanism does not appear to be common in the modern universe. It is likely that the pathways discussed above are not an exhaustive sample; therefore, creating a large sample of galaxies undergoing this transformation is necessary to probe the various conditions that can trigger it, possibly identifying new pathways that lead a galaxy to evolve.

Despite the color and morphology bimodalities, finding galaxies that are rapidly transitioning is more complicated than determining their colors and morphologies. Schawinski et al. (2014) showed that the number of galaxies within the green valley undergoing morphological change is small compared to galaxies whose intermediate colors are caused by secular processes, in which normal spiral galaxies with normal starforming histories build up a substantial population of lowermass (redder) stars with a constant star formation rate, gradually turning the integrated colors of the galaxy green.

More recently, a mid-infrared (mid-IR) color bimodality was observed using the Wide-field Infrared Survey Explorer (WISE; Wright et al. 2010). Authors identified a bimodality in both the [3.4]-[12] $\mu \mathrm{m}$ (Ko et al. 2013, 2016) as well as the [4.6][12] $\mu \mathrm{m}$ colors (Alatalo et al. 2014a; Yesuf et al. 2014). In the case of the [4.6]-[12] colors, Alatalo et al. (2014a) showed that color bimodality not only split based on galaxy morphology but that it was also more prominent than optical colors, and named it the "infrared transition zone" (IRTZ).

Post-starburst galaxies are one such example that has robustly been shown to have undergone a rapid cessation of star formation (Dressler \& Gunn 1983; Zabludoff et al. 1996) via the presence of stellar absorption features consistent with intermediate stellar populations (such as strong Balmer absorption; Vazdekis et al. 2010) and a lack of nebular ionized gas emission, such as $\mathrm{H} \alpha$ or $[\mathrm{O} \mathrm{II}] \lambda 3727$, which originates from $\mathrm{H}$ II regions associated with current (within the last $10 \mathrm{Myr}$ ) star formation. These methods include the " $\mathrm{K}+\mathrm{A}$ " method, which uses a weighting of A-star and K-star stellar libraries to determine a young star fraction (Dressler \& Gunn 1983; Quintero et al. 2004) or a "E+A" identification (an early-type galaxy with A-type stars), which relies on Balmer absorption identification (Goto 2005, 2007). Although it is likely that the stringent selections used to pinpoint post-starburst galaxies miss a non-negligible fraction of transitioning galaxies, including those that host quasars (Canalizo \& Stockton 2000, 2013; Cales et al. 2011, 2013; Cales \& Brotherton 2015) or shocks (Davis et al. 2012; Alatalo et al. 2014b, 2014a, 2016a), they are a bona fide sample of transitioning galaxies.

We utilize the post-starburst sample compiled by Goto (2007) to probe various properties of transitioning galaxies, including whether the WISE colors and the IRTZ (Alatalo et al. 2014a) are able to identify a galaxy as having recently undergone a transformation. Given that post-starburst identification relies on available spectroscopy, being able to use photometry alone to pinpoint transitioning galaxies has the potential to substantially increase the total number of galaxies identified as undergoing this metamorphosis.

The paper is organized as follows. In Section 2, we describe our post-starburst sample selection and comparison sample. In Section 3, we describe the post-starburst WISE properties and interpret those results. In Section 4, we summarize our findings. The cosmological parameters $H_{0}=70 \mathrm{~km} \mathrm{~s}^{-1}, \Omega_{m}=0.3$, and $\Omega_{\Lambda}=0.7$ (Spergel et al. 2007) are used throughout.

\section{Results and Analysis}

\subsection{Sample Selection}

We used the post-starburst galaxy sample defined by Goto (2007) of 564 galaxies from the Sloan Digital Sky Survey Data Release 5 (SDSS DR5; Adelman-McCarthy et al. 2007), selected using the "E+A" criterion of deep Balmer absorption (EW $(\mathrm{H} \delta)>5 \AA$ ) combined with weak nebular (EW H $\alpha<3 \AA$, and EW $[\mathrm{O}$ II $]<2.5 \AA$ ) emission. These objects have redshifts ranging between 0.03-0.34. We cross-matched this sample with the WISE catalog (Wright et al. 2010) and SDSS Data Release 9 (DR9; Ahn et al. 2012), using TOPCAT (Taylor 2005). Of the original 564 post-starburst galaxies, 560 have robust $(\mathrm{S} / \mathrm{N}>3)$ detections in the $\mathrm{W} 1 / 3.4 \mu \mathrm{m}, \mathrm{W} 2 / 4.6 \mu \mathrm{m}$, and $\mathrm{W} 3 / 12 \mu \mathrm{m}$ bands. In the $u, r$, and $i$ filters, 534 objects are detected robustly.

In most cases, we used the profile fit ( $\mathrm{W} \star \mathrm{mprO}$ ) value from the WISE All-sky catalog for the WISE colors. When objects were flagged as extended, we elected to use $\mathrm{w} \star$ gmag, which is the value derived using the 2-Micron All-Sky Survey (2MASS; Skrutskie et al. 2006) profile fit, for the same aperture. The $u-r$ colors are $k$-corrected using the calc_kcor IDL routine (Chilingarian \& Zolotukhin 2012). ${ }^{18}$

\footnotetext{
18 http://kcor.sai.msu.ru/
} 
In order to obtain a robust sub-selection of objects with near-IR data for a complete spectral energy distribution (SED), we cross-matched the 564 post-starburst galaxies from Goto (2007) with the 2MASS Extended Source Catalog (XSC; Skrutskie et al. 2006) containing the extended source photometries of 1.7 million galaxies. ${ }^{19}$ In doing so, we recovered extended source photometries for 190 post-starburst galaxies. Then we cross-matched with the full-photometry catalogs of these samples (containing both SDSS and WISE data). Of the 190 post-starbursts, 158 were detected in the WISE3.4, 4.6, and $12 \mu \mathrm{m}$ bands. Only 53 of the post-starbursts were detected in the WISE $22 \mu \mathrm{m}^{20}$ band with $\mathrm{S} / \mathrm{N}>3$. For subsequent color plots, we use the $534 \mathrm{E}+\mathrm{A}$ galaxies robustly detected in the SDSS $u, r$, and $i$, bands and WISE W1, W2, and W3 bands. For subsequent composite SED plots, we use the 190 XSC E+A galaxies.

For our comparison sample, we use the morphologically classified Galaxy Zoo (Lintott et al. 2008) objects from Schawinski et al. (2014). We also cross-matched these 47,995 Galaxy Zoo objects with the 2MASS XSC, resulting in 38,802 Galaxy Zoo matches. The Galaxy Zoo comparison sample was drawn from Schawinski et al. (2014) and Alatalo et al. (2014a), and a corresponding analysis of the derivation of WISE colors can be found therein. Figures 1 and 2 show the optical and WISE color distributions of both Galaxy Zoo and the post-starbursts.

\subsection{Redshift Dependence of the WISE Colors}

Figure 3 shows a significant deviation of the [3.4]-[4.6] WISE colors in post-starburst galaxies. Upon closer inspection, these colors have a substantial dependence on the redshift of the source, with the most significantly red colors having the highest redshifts. Brown et al. (2014) showed that, given that the SEDs of many galaxies go from decreasing to increasing in the mid-IR, accounting for a redshift dependence can be important. Figure 4 shows the [3.4]-[4.6] and [4.6]-[12] colors for the post-starburst sample versus redshift, in both cases showing dependences. This is clear both in the individual post-starburst colors, as well as in the average colors in redshift bins. A slight redshift dependence is also seen in the [4.6]-[12] colors, though the trend is smaller than the scatter in each bin. A significant redshift dependence in the [3.4]-[4.6] colors is seen, with a marked increase followed by a flattening at $z \geqslant 0.2$.

There are many possible causes for this dependence, including the effects of aperture bias, Malmquist bias (Malmquist 1925), and and redshifting the SED. It is possible that the type of post-starburst galaxy that the (Goto 2007) criterion selected could have changed between the lowredshift objects, where the SDSS fiber subtends a smaller fraction of the galaxy and the higher-redshift objects, where much more of the galaxy is sampled. It is also possible that the Goto (2007) selection detects brighter, rarer objects at higher redshifts. But given that the mid-IR is the location at which the SED transitions between the Rayleigh-Jeans tail of the stellar light of the galaxy and the hot dust component originating in the circumstellar envelopes of aging stars and

\footnotetext{
19 http://www.ipac.caltech.edu/2mass/releases/allsky/doc/sec2_3.html

${ }^{20}$ The updated filter response function of the WISE W4 band places the central wavelength closer to $23 \mu \mathrm{m}$ (Brown et al. 2014), but we use $22 \mu \mathrm{m}$ for consistency.
}

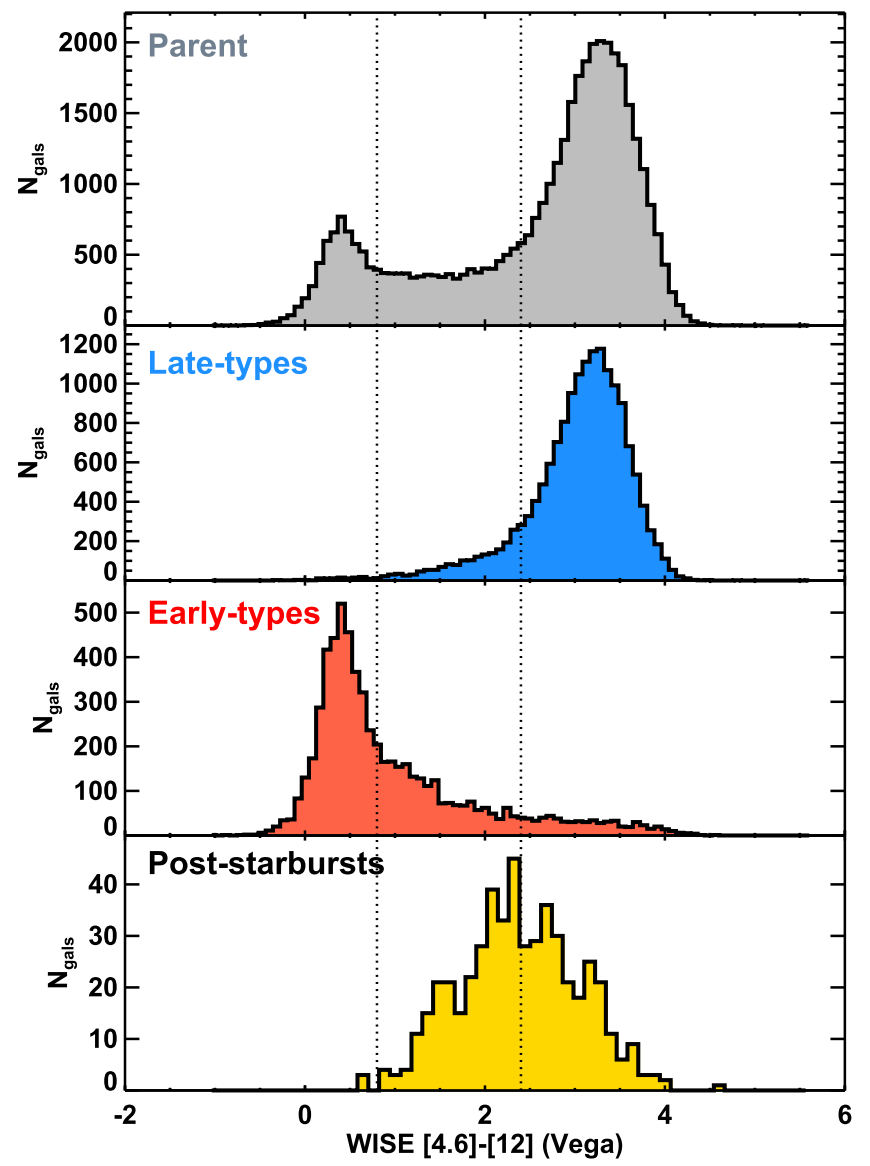

Figure 1. [4.6]-[12] WISE color distributions of the various samples, including the Galaxy Zoo comparison sample of early-type and late-type galaxies (Schawinski et al. 2014; Alatalo et al. 2014a; gray, top), delineated between late-type galaxies (blue) and early-type galaxies (red), with redshift-corrected post-starburst galaxy colors for comparison (yellow). The boundaries of the IRTZ are shown as a striped line through all plots. Post-starburst galaxies peak on the star-forming side of the IRTZ, and have the highest fractional IRTZ representation of the galaxies shown.

shrouded star formation (Silva et al. 1998), it is likely that this has the most dramatic effect on the [3.4]-[4.6] colors. A comprehensive SED fit to these galaxies is required to fully understand how each of these biases might impact our sample, and the constituents to the SED (discussed in Section 3.2) makes applying accurate $k$-corrections difficult.

To provide corrections to the [3.4]-[4.6] and [4.6]-[12] colors, we binned the post-starburst galaxies by redshift, every $z=0.03$, from 0.03 to 0.3 with $\Delta z=0.03$. The average color of each bin was then determined for the colors, shown as the black squares in Figure 4. The standard deviation of each redshift bin is reflected in the error bars. The Galaxy Zoo sample (Alatalo et al. 2014a; Schawinski et al. 2014) consists of objects with redshifts between $z=0.02-0.05$. In order to reflect an accurate comparison to Galaxy Zoo, we "correct" the [3.4]-[4.6] and [4.6]-[12] colors of the post-starbursts by normalizing to the $z=0.03-0.06$ bin. We determine the redshift bin that each individual post-starburst galaxy sits in, then subtract the difference between the average color of that redshift bin from the average color of the $z=0.03-0.06$ bin. Figures 1 and 2 use WISE colors that have been corrected this way. 

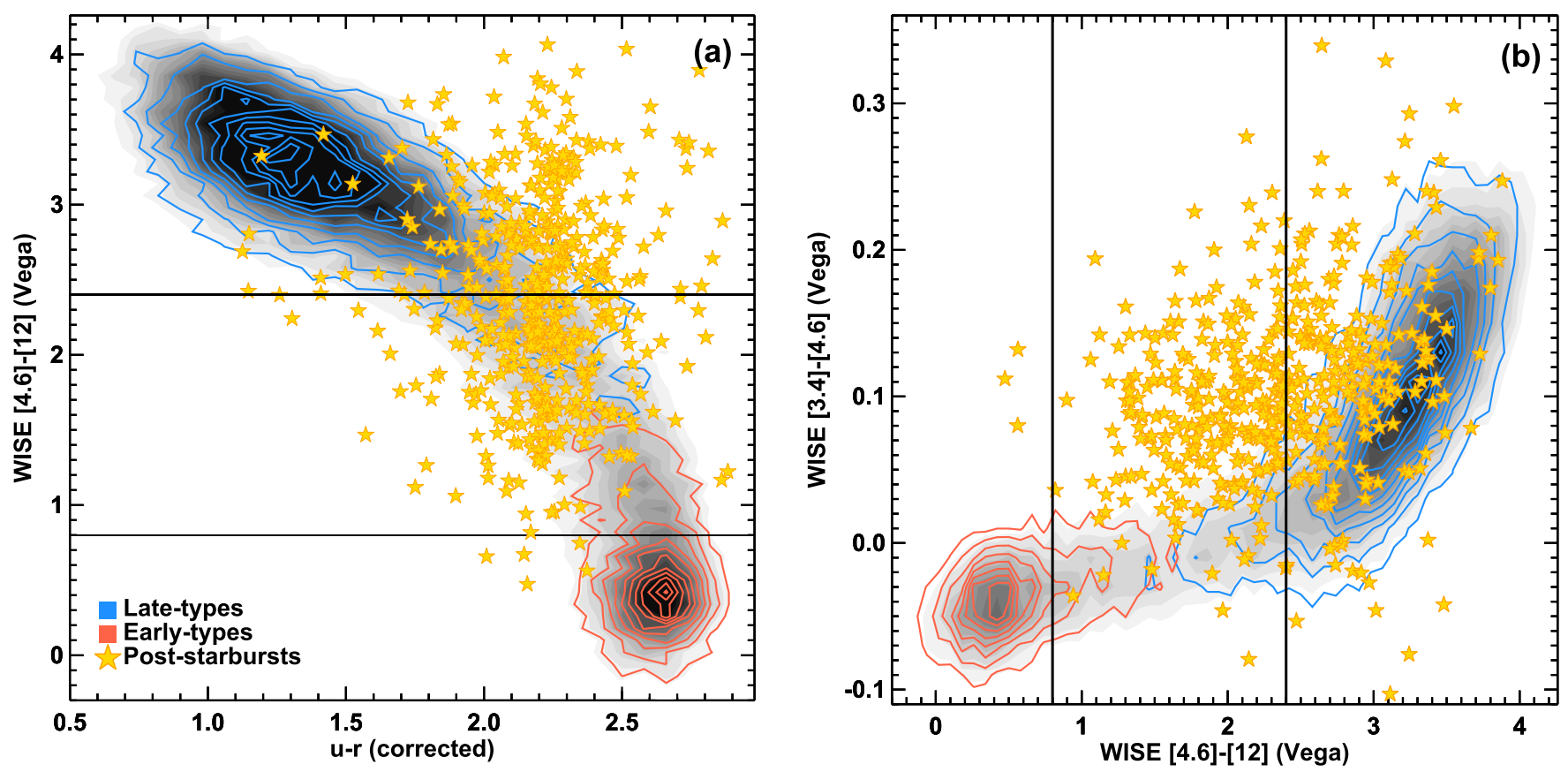

Figure 2. (Left) $u-r$ vs. redshift-corrected [4.6]-[12] colors show a strong correlation with the prominent separation between late-types (blue contours) and early-types (red contours) from Schawinski et al. (2014). Post-starbursts (yellow stars) are located right between the two populations. (Right) [4.6]-[12] vs. [3.4]-[4.6] WISE colors, which show that the post-starburst galaxies have slightly elevated [3.4]-[4.6] colors compared to the Galaxy Zoo sample. Post-starbursts have colors that are consistent with the Seyfert sample shown in Alatalo et al. (2014a). The IRTZ is shown as black solid lines in each plot. The optical colors are $k$-corrected. The mid-IR colors are redshift-corrected (see Section 2.2).

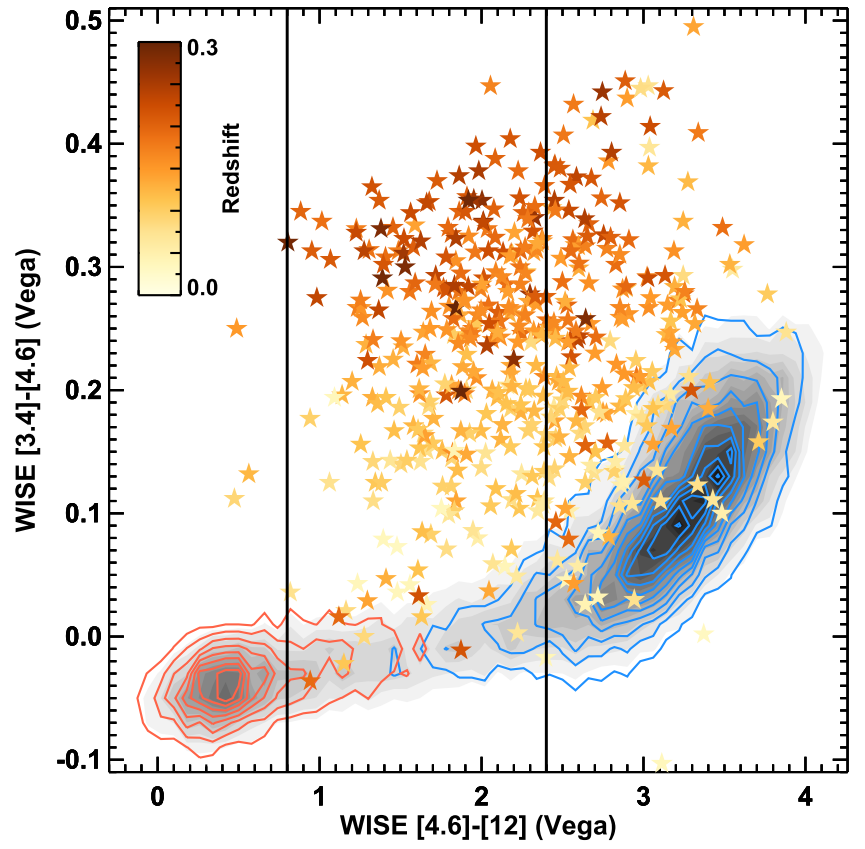

Figure 3. [4.6]-[12] vs. [3.4]-[4.6] WISE colors of post-starbursts (stars) as compared to the early-type (red contours) and late-type (blue contours) galaxies from Schawinski et al. (2014) and Alatalo et al. (2014a). The redshifts of the post-starbursts are encoded using the color scale on the upper left. A redshift dependence of the [3.4]-[4.6] colors is clear, with the most elevated colors coming from the highest-redshift sources.

\section{Discussion}

\subsection{The WISE Colors of Post-starburst Galaxies}

Figure 1 shows the distribution of the [4.6]-[12] colors of the Galaxy Zoo sample (Alatalo et al. 2014a; Schawinski et al. 2014), separated into the late-type and early-type subsamples with the WISE IRTZ overplotted. As was shown in Alatalo et al. (2014a), the Galaxy Zoo samples show a bimodal distribution, with a zone of avoidance between the late-type and early-type populations. The post-starburst sample is strongly represented within the IRTZ, with $47.6 \pm 2.2 \%$ falling within the bounds set in Alatalo et al. (2014a) (compared with $16.1 \pm 0.2 \%$ of the Galaxy Zoo sample, $22.7 \pm 0.4 \%$ of early-types, and $10.5 \pm 0.2 \%$ of the late-types), consistent with the hypothesis that the IRTZ is able to pinpoint galaxies that are transitioning. The Mann-Whitney U test (IDL routine RS_test) was run to compare each pair of [4.6]-[12] WISE color distributions, which confirmed that post-starburst galaxies are a distinct population with a $p$ value $\ll 10^{-5}$ in all cases.

Figure 2 further supports this picture, placing post-starburst galaxies firmly within the transitioning region. Post-starbursts are located primarily in the optical green valley (Dressler \& Gunn 1983) and also appear in the WISE IRTZ. The poststarburst population most obviously falls into the transition zone when viewed in $u-r$ versus WISE [4.6]-[12] color space, positioned among the tight color correlation between the earlytype and late-type populations (Figure 2(a)). The post-starburst colors confirm that the WISE IRTZ traces a transitioning population and can be used as part of a criterion to identify galaxies through their photometry.

Post-starburst galaxies separate themselves into an elevated [3.4]-[4.6] versus [4.6]-[12] $\mu \mathrm{m}$ WISE color space (Figure 2(b); also see Figure 15 in Yesuf et al. 2014 for the colors of a similar sample). $48 \pm 2 \%$ of galaxies fall outside of the $10 \%$ contours of the Galaxy Zoo sample, and $91 \pm 1 \%$ fall outside the $50 \%$ contours of the Galaxy Zoo sample. These colors seem to indicate that post-starbursts do not traverse WISE color space through the joint between the early-type and late-types, rather showing signs of elevated [3.4]-[4.6] colors (and intermediate 

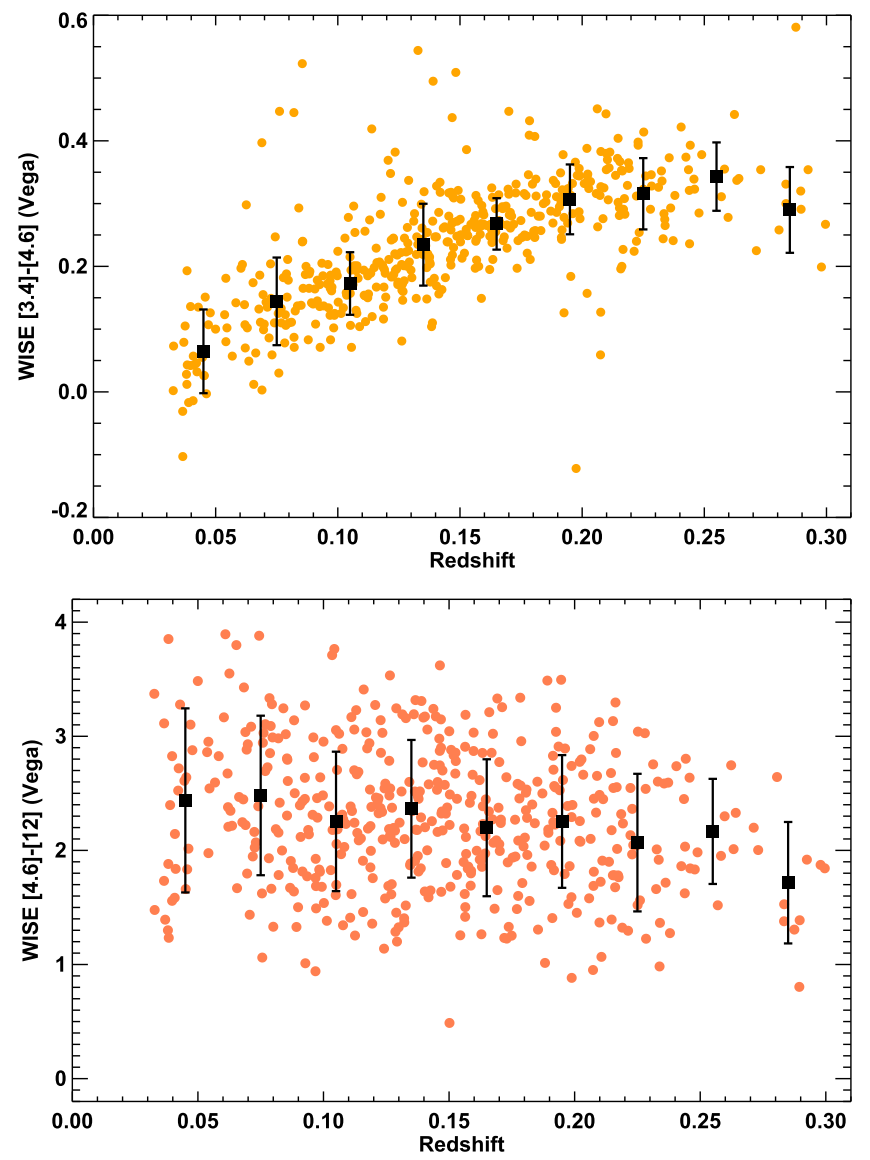

Figure 4. (Top) [3.4]-[4.6] colors of each of the 560 WISE-detected poststarbursts (orange points) vs. redshift. The points are then binned in redshift, spaced in bins of $z=0.03$, ranging from $z=0.03-0.3$, with $\Delta z=0.03$. The black squares represent the average [3.4]-[4.6] colors, with the error bars representing their standard deviation. A clear redshift dependence is seen in these colors that is more substantial than the scatter in the bins. (Bottom) [4.6]-[12] colors of each of the 560 WISE-detected post-starbursts (red points) vs. redshift. The black squares represent the average colors in redshift bins, with the error bars representing the standard deviation. In this case, although there might be a slight trend with redshift, it is well within the (significant) scatter.

[4.6]-[12] colors) and traversing through a mid-IR "twilight zone." The zone could be a consequence of the continued presence of ongoing star formation (Peletier et al. 2012; Hayward et al. 2014), or the presence of an AGN (van der Wolk 2011; Assef et al. 2013; Mateos et al. 2015).

\subsection{The Origin of Post-starburst WISE Colors}

To further investigate the mid-IR twilight zone, we construct the average SEDs of the post-starbursts, early-types, and latetypes. Figure 5 shows the SEDs of each of these subsamples, with the top and bottom of the bar representing the upper and lower quartiles, respectively. The point represents the median value for each band. Early-type galaxies are generally redder than late-type galaxies, and post-starbursts generally fall in between. In the WISE bands, the post-starbursts exhibit significantly stronger emission at [3.4] and [4.6] microns, as well as a shallower gradient. The $12 \mu \mathrm{m}$ flux is generally in between that of the late-type and early-types, with much more overlap with the late-type galaxies.

Figure 6 separates the SEDs of the post-starburst galaxies with (53) or without (108) detected $22 \mu \mathrm{m}$ emission. We created a composite SED (with the bars representing the upper and lower quartiles of the distribution, the point representing the median, and the error bars representing the standard deviation of the median) of the $22 \mu$ m-detected objects, shown in the first panel of Figure 6. The 12 and $22 \mu \mathrm{m}$ SEDs show a leveling off or increasing SED that cannot be reproduced using stars alone (Bruzual \& Charlot 2003). While a compact starburst is capable of creating this rise in the SED, we consider this possibility unlikely. The compact starburst hypothesis assumes that the $\mathrm{E}+\mathrm{A}$ selection is unable to remove these sources. Given that gas and extreme star formation coalesce into the nucleus of post-merged galaxies (Mihos \& Hernquist 1996; Bryant \& Scoville 1999), it is unlikely that the SDSS spectrum would contain so little $\mathrm{H} \alpha$ emission that it would make it through the $\mathrm{E}+\mathrm{A}$ cut. Additionally, these buried compact starbursts are inconsistent with the $1.4 \mathrm{GHz}$ radio continuum results in $\mathrm{K}+\mathrm{A}$ galaxies (Nielsen et al. 2012).

The $22 \mu \mathrm{m}$ emission in post-starbursts not individually detected significantly by WISE were stacked to create a composite image. Each $22 \mu \mathrm{m}$ thumbnail was downloaded from the ALLWISE ${ }^{21}$ catalog (Wright et al. 2010). The poststarburst source was placed in the center, and the stacked image was created by taking the average of stacked pixels ( $4 \sigma$ outliers in each pixel were clipped to remove contributions from bright stars in individual fields), resulting in a $22 \mu \mathrm{m}$ detection from the stack. To extract the photometry of the stacked $22 \mu \mathrm{m}$ emission, we used an aperture with a radius of $24^{\prime \prime}$ (i.e., twice the WISE22 $\mu \mathrm{m}$ resolution). We then subtracted the median emission from the "sky" in an annulus between $35^{\prime \prime}<r<55^{\prime}$. The resulting photometry (calculated and converted using the WISE manual ${ }^{22}$ ) is: $m=15.86_{-0.25}^{+0.32}$ in $\mathrm{AB}$ magnitudes.

The photometry of each of the 190 XSC post-starburst galaxies was fit using MAGPHYS (da Cunha et al. 2008), which is described in Appendix A. We isolate the MAGPHYS-derived stellar and hot $\left(T_{\text {dust }}=130-250 \mathrm{~K}\right)$ dust luminosity components for each post-starburst, and compare them to early-types, spirals, and AGNs. ${ }^{23}$ We find that the [4.6]-[12] color from WISE correlates with, and is therefore a good proxy for, the ratio of the luminosities of the hot dust and stellar emission. This is somewhat expected, as the $4.6 \mu \mathrm{m}$ emission primarily traces the stellar blackbody emission and the $12 \mu \mathrm{m}$ emission primarily traces hot dust (from either star formation, aged stars, or AGNs).

Figure 7 also shows that the post-starbursts have middling luminosity ratios, between early-types and spirals/AGNs. Poststarbursts are expected to be in a transitional state between spirals and early-type galaxies (or possibly rejuvenated earlytype galaxies; Dressler et al. 2013; Abramson et al. 2014), so perhaps their intermediate luminosities are unsurprising. This conclusion, however, assumes that the hot dust emission has a similar origin to the hot dust emission in spirals (i.e., star formation). Given that the very selection of these galaxies precludes star formation from being significant, it is likely that the hot dust luminosity (and additionally the mid-IR emission from WISE) has an alternative heating mechanism. We lay out these possibilities in subsequent sections.

\footnotetext{
21 http://wise2.ipac.caltech.edu/docs/release/allwise/

$22 \mathrm{http} / /$ wise2.ipac.caltech.edu/docs/release/allsky/expsup/sec4_4c.html

${ }^{23}$ Details on the comparison samples are discussed in Appendix B.
} 


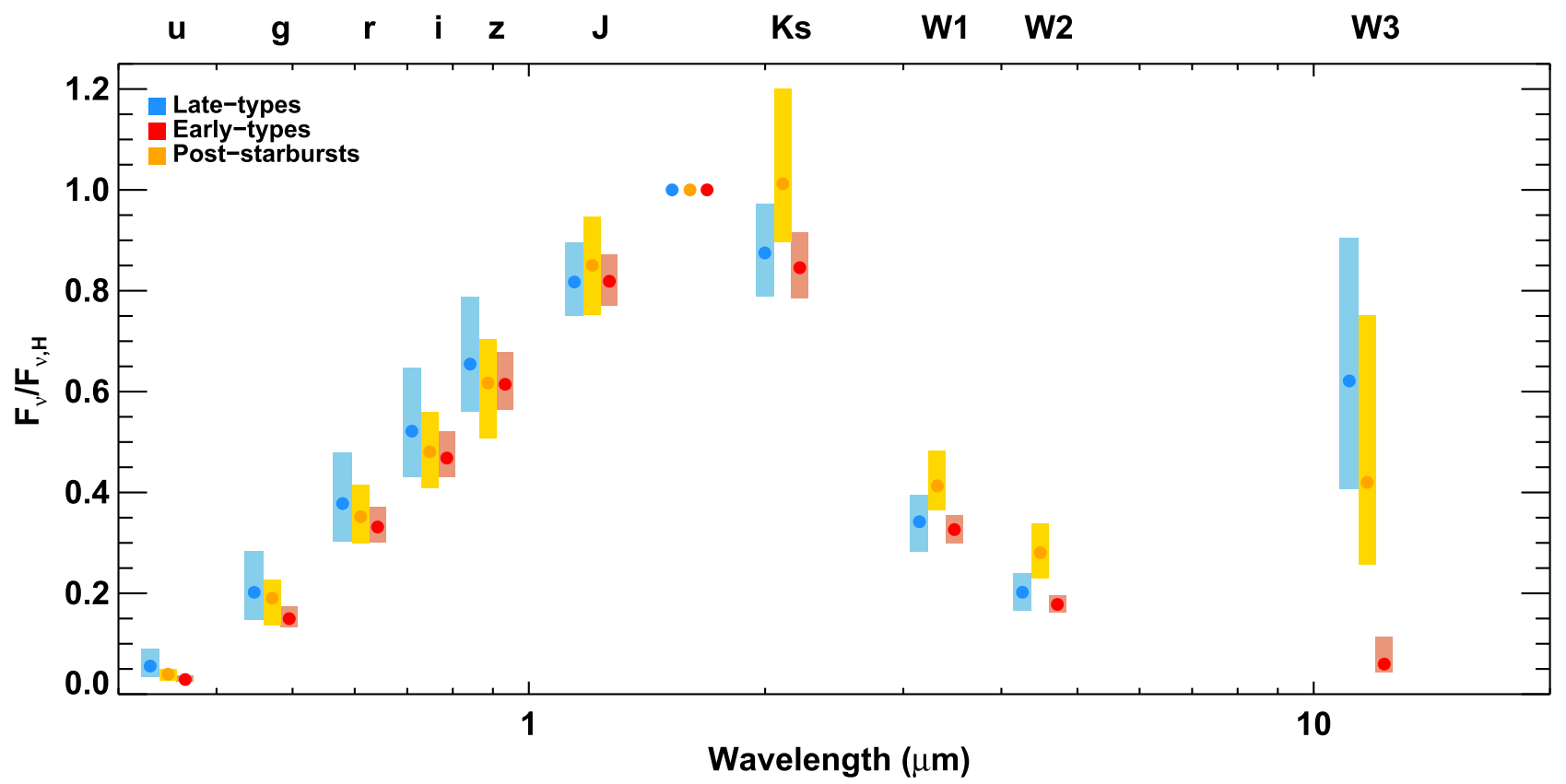

Figure 5. The $u$ g r $i$ zJ H K plus WISE band 123 observed frame SEDs of each subsample: early-types (red), late-types (blue), and the 158 extended post-starbursts (yellow), normalized to their $H$-band fluxes. The width of the bar represents the upper and lower quartiles, and the point is placed at the median value for each band. Post-starbursts contain colors that are in between early-type and late-type galaxies. Post-starbursts also show significant emission in 3.4 and $4.6 \mu$ m, with a shallower gradient. The $12 \mu \mathrm{m}$ flux in post-starbursts lies in between the late-type and early-type $12 \mu \mathrm{m}$ fluxes, with much more overlap with the late-type galaxies.

\subsubsection{PAHs in Post-starbursts}

In normal star-forming galaxies, significant emission from photospheres and warm dust and polycyclic aromatic hydrocarbons (PAHs) in the interstellar medium (Calzetti et al. 2007; Smith et al. 2007) usually overwhelms the mid-IR SED. Thus, in most star-forming galaxies, the WISE 12 and $22 \mu \mathrm{m}$ bands are detecting active, recent star formation. In extreme starbursts (such as M82), there is a significant compact, hot dust component, leading to a rising SED between 12 and $22 \mu \mathrm{m}$ (Sturm et al. 2000; Beirão et al. 2008). Post-starbursts selected by Goto (2007) removed all objects with significant $\mathrm{H} \alpha$ emission; thus it is unsurprising that their SEDs do not match those of the star-forming galaxies in Figure 5, but the enhanced $12 \mu \mathrm{m}$ emission (as compared to early-types) is worth discussing further.

By the time the galaxies have quenched and become completely quiescent, the total number of evolved intermediate age stars has diminished. Therefore, the mid-IR emission in quiescent galaxies likely originates from a diffuse dust component (Temi et al. 2007; Boselli et al. 2010; Ciesla et al. 2014), though usually the dust emission is dwarfed by the stellar emission. PAH emission is detectable in early-type galaxies (Xilouris et al. 2004; Kaneda et al. 2005, 2008; Bressan et al. 2006; Panuzzo et al. 2007; Bregman et al. 2008) and is significant in $\mathrm{K}+\mathrm{A}$ galaxies (Roseboom et al. 2009). In both of these cases, the $12 \mu \mathrm{m}$ emission is specifically enhanced due to the $11.3 \mu \mathrm{m}$ neutral $\mathrm{PAH}$ feature, which could explain the significant $12 \mu \mathrm{m}$ emission we see in Figure 5. This was argued to be part of the existence of the IRTZ by Alatalo et al. (2014a).

Vega et al. (2010) presented the possibility that the unusual neutral-to-ionized PAH ratios that are observed are not due to accreted gas or an AGN but are instead due to the processing of carbonaceous material from the circumstellar envelopes of thermally pulsating asymptotic giant branch (TP-AGB) stars combined with slow shocks. The combination of these two processes may be able to create amorphous carbon and destroy the smaller PAHs (that create the shorter wavelength ionized PAH bands), leading to the enhanced $11.3 \mu \mathrm{m}$ neutral emission. Thus, it is possible that $11.3 \mu \mathrm{m}$ PAH emission (a potential source for the enhanced $12 \mu \mathrm{m}$ emission) could originate from TP-AGB stars.

\subsubsection{AGB Stars in Post-starbursts}

Figure 8 presents the [4.6]-[12] versus [12]-[22] WISE colors for oxygen-rich asymptotic giant branch (AGB) stars (Suh \& Kwon 2011), Stripe $82^{24}$ "strong" $\mathrm{AGNs}^{25}$ (blue points; E. Glikman et al. 2017, in preparation), $22 \mu \mathrm{m}$ nondetected (dark orange triangles), and $22 \mu \mathrm{m}$-detected poststarbursts (yellow stars). For many of the post-starbursts (both $22 \mu$ m-detected and non-detected), the [4.6]-[12] colors are consistent with oxygen-rich AGB stars, whose contributions to the optical spectra peak during the post-starburst phase of a galaxy (Yan et al. 2006), and are inconsistent with the normal star-forming population (as is seen in the [4.6]-[12] color comparison in Figure 2).

Post-starburst galaxies are an ideal population to study midIR emission from the AGB population, given that their star formation has been quenched and no longer contributes, and the intermediate age stars are still abundant. Emission from circumstellar dust shells originating in TP-AGB stars tend to peak in the mid-IR (Piovan et al. 2003; Maraston 2005; Kelson \& Holden 2010; Chisari \& Kelson 2012). A 2 Gyr old AGB component leads to a slightly shallower slope in the mid-IR portion of the SED, which is observed in the post-starburst composite SED in Figure 5.

\footnotetext{
24 http://classic.sdss.org/legacy/stripe82.html

25 AGNs that are brighter than their hosts' starlight.
} 


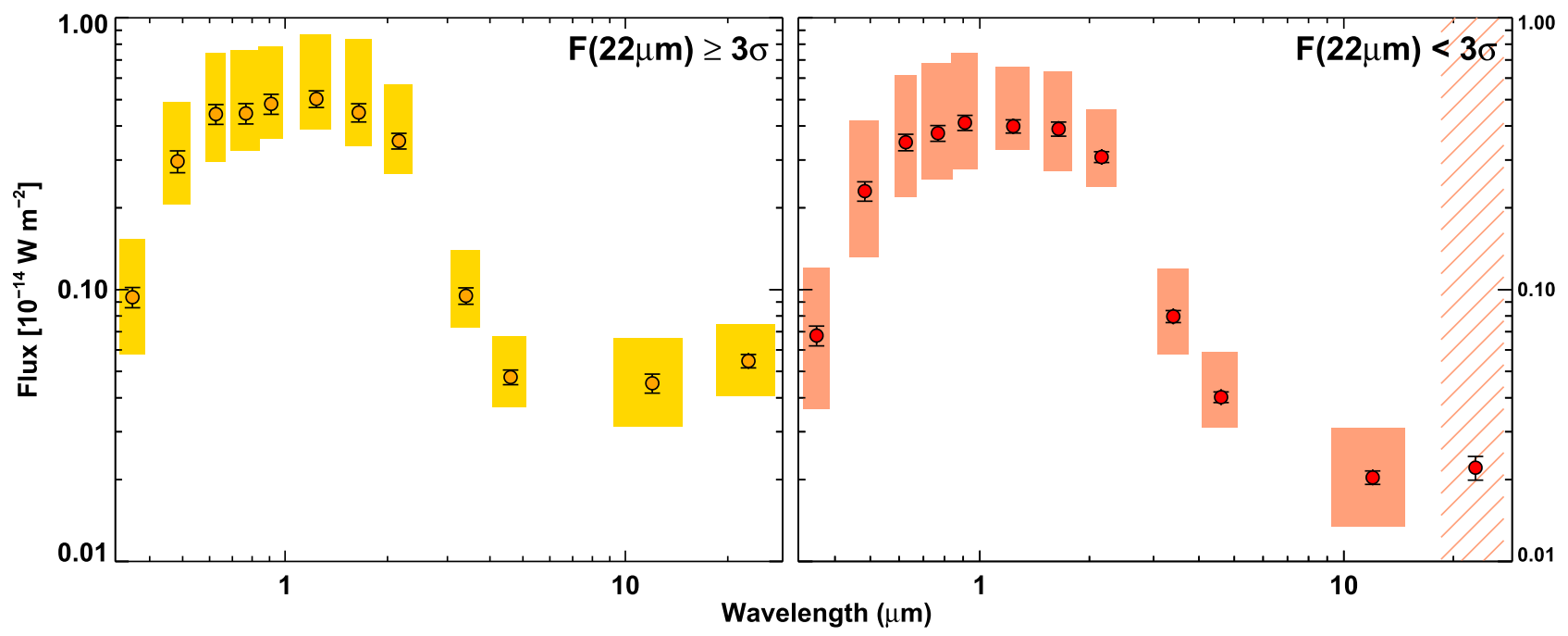

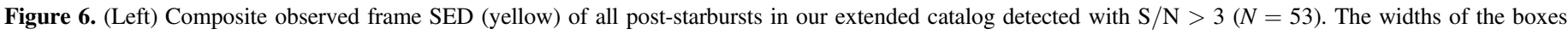

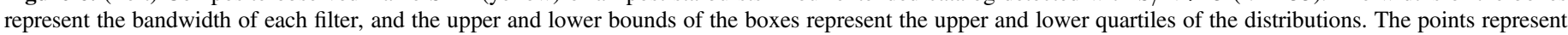

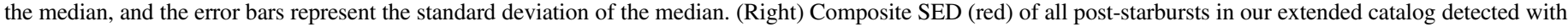

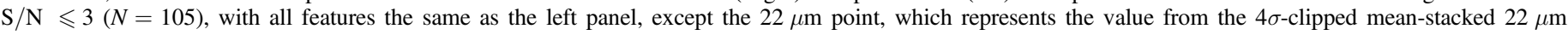

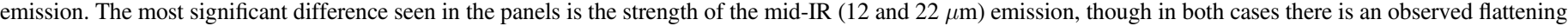
in the mid-IR SED.

Previous studies of the TP-AGB and post-AGB contributions to post-starbursts have been contradictory, with optical spectra suggestive of their contribution (Yan et al. 2006) but IR spectra (Zibetti et al. 2013) and SED studies inconsistent with a dominant AGB component (Kriek et al. 2010; Melnick \& De Propris 2013). In the cases of contrasting studies, many used models that required a "heavy" AGB contribution that did not include circumstellar dust (Maraston 2005) and did not fit the SED out to the mid-IR. When mid-IR data are included, and the AGB model is modified to include circumstellar dust, TP-AGB and post-AGB models more consistently match the SEDs (Maraston et al. 2013). The [4.6]-[12] colors from Figure 8 seem to indicate that these stars could contribute to the mid-IR SED of post-starbursts, but the data are inconclusive. Thus, it is not clear what impact TP-AGB and post-AGB stars have on the integrated light of post-starburst galaxies; it will require further study to disentangle from other contributors to the $12 \mu \mathrm{m}$ portion of a galaxy's SED.

\subsubsection{AGNs in Post-starbursts}

The WISE [3.4]-[4.6] versus [4.6]-[12] colors of poststarburst galaxies shown in Figure 2(b) suggest that the transition in infrared color space of these objects is not a simple pathway in color space across the boundary that separates the early-type and late-type galaxy distributions. A robust exploration of the physical processes taking place in the quenching galaxies, and how those manifest in their integrated properties, can shed light on the way in which post-starburst galaxies undergo their metamorphosis.

Post-starburst galaxies are thought to be the final stage of a transitioning galaxy. Evidence exists that there is a delay between star formation quenching and the onset of AGN activity (Canalizo \& Stockton 2001; Schawinski et al. 2007; Kaviraj et al. 2015; Matsuoka et al. 2015; Bitsakis et al. 2016); thus, we might expect post-starburst galaxies to disproportionately host AGNs, assuming that they are just post-transition. The distribution of post-starburst WISE colors in fact show similarities to the Seyfert population discussed in Alatalo et al. (2014a). Could this be a sign that the WISE colors of post-starburst galaxies originate from a buried AGN component?

Many studies of post-starbursts have aimed to confirm the presence of AGNs in these systems. Brown et al. (2009) observed a slight enhancement in X-rays in a sample of $\mathrm{K}+\mathrm{A}$ galaxies in the NOAO Deep Wide-field Survey, though not to a significance to definitively identify it as originating from AGN emission. Shin et al. (2011) cross-identify $1.4 \mathrm{GHz}$ FIRST (Becker et al. 1995) sources with the Goto (2007) E+A sample, but detections are ambiguous (most sources are unresolved and lacking clear radio jets). Nielsen et al. (2012) cross-referenced $\mathrm{K}+\mathrm{A}$ galaxies with FIRST (Becker et al. 1995), and noted enhanced radio emission in some of them, which could be attributed to either AGN activity or remnant star formation. Though all of these studies could be pointing to low-luminosity AGNs being present, none are definitive. Meusinger et al. (2017) studied a large sample of $\mathrm{E}+\mathrm{A}$ galaxies, finding a slightly elevated number of luminous $\left(>10^{23} \mathrm{~W}\right) 1.4 \mathrm{GHz}$ continuum sources and an enhanced fraction of mid-IR WISEselected (Assef et al. 2013) AGNs, and confirmed the rising mid-IR SED discussed by Melnick \& De Propris (2013), but concluded that while $\mathrm{E}+\mathrm{A}$ galaxies do not host strong AGNs, they may contain obscured and/or low-luminosity AGNs or require significant emission from post-AGB stars (as discussed in Section 3.2.2).

The right panel of Figure 6 shows the composite SED created from the 105 WISE $22 \mu \mathrm{m}$ non-detected objects, with quartiles and medians from data points up to $12 \mu \mathrm{m}$, consistent with the right panel. In these objects, there is a more significant drop between the $4.6 \mu \mathrm{m}$ data point and the $12 \mu \mathrm{m}$, but there is still observed flattening in the mid-IR.

The $22 \mu \mathrm{m}$ emission can also be produced by remnant hot dust emission from the recently quenched episode of star formation, which has been known to cause the overestimation of star formation in these types of sources (Hayward et al. 2014; Utomo et al. 2014). Sources that show these overestimates often show a decrease between the 12 and $22 \mu \mathrm{m}$ bands, which is not the behavior that we observe in our sample 


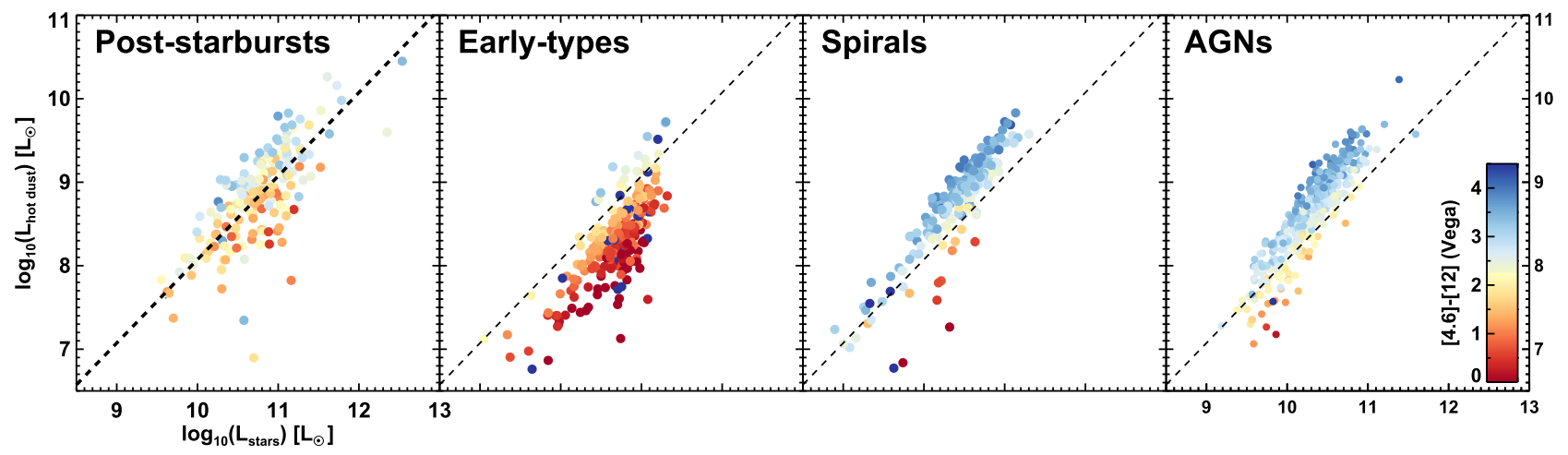

Figure 7. The MAGPHYS-derived stellar vs. hot dust luminosity, color-coded based on the WISE [4.6]-[12] $\mu$ m colors of post-starbursts (left), early-types galaxies (middle left), spirals (middle right), and AGNs (right). The stellar luminosity range is the same in all panels. The dashed black line corresponds to the mean relationship between the stellar and hot dust luminosities for the post-starbursts in all panels. The WISE [4.6]-[12] $\mu \mathrm{m}$ colors are correlated with the relative strength of the hot dust (compared to stellar) luminosities. Early-type galaxies are the most stellar luminosity-dominant, and both spirals and AGNs have larger hot dust (compared to stellar) luminosities, though it is likely that the hot dust emission originates from a different source for each. Post-starbursts have hot dust-to-stellar luminosities that are between the early-type and spiral or AGN values.

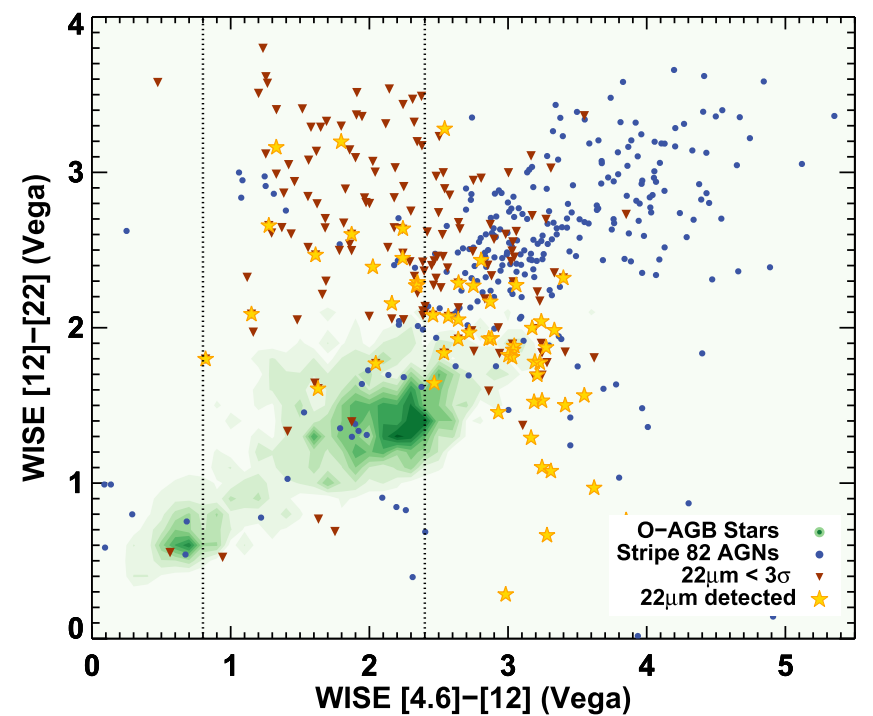

Figure 8. [4.6]-[12] vs. [12]-[22] WISE colors for oxygen-rich AGB stars (green colorscale; Suh \& Kwon 2011), Stripe 82 "strong" AGNs (blue points; E. Glikman et al. 2017, in preparation), $22 \mu \mathrm{m}$ non-detected (dark triangles), and $22 \mu \mathrm{m}$-detected post-starbursts (yellow stars) from the extended source sample of 158 . The IRTZ is represented by black dotted lines. As was shown in Figures 1 and 2, post-starbursts fall in the infrared transition zone. Many poststarbursts exhibit [12]-[22] colors consistent with AGNs.

in Figure 6. Alternative processes able to create a rising mid-IR SED at wavelengths shorter than $10 \mu \mathrm{m}$ and provide sufficient emission to balance the stellar light from the galaxy are starbursts or AGNs. Starbursts have been ruled out by the weak nebular emission in the post-starburst systems (Goto 2007), especially since the majority of post-merger star formation activity is expected to occur in the nucleus (Mihos \& Hernquist 1996), at the position of the SDSS spectral fiber. Figure 9 shows the 2-30 $\mu \mathrm{m}$ ranges of five phenomena that contribute to the mid-IR SED, including stellar emission (Bruzual \& Charlot 2003), AGB stars (Piovan et al. 2003), star formation, (including PAH emission; Brown et al. 2014), a starburst galaxy (Mrk 33; Brown et al. 2014), and hot dust from an AGN (Sajina et al. 2012). Both the starburst and AGN hot dust templates exhibit rising mid-IR emission, but it is improbable that the majority of post-starburst galaxies contain buried H II

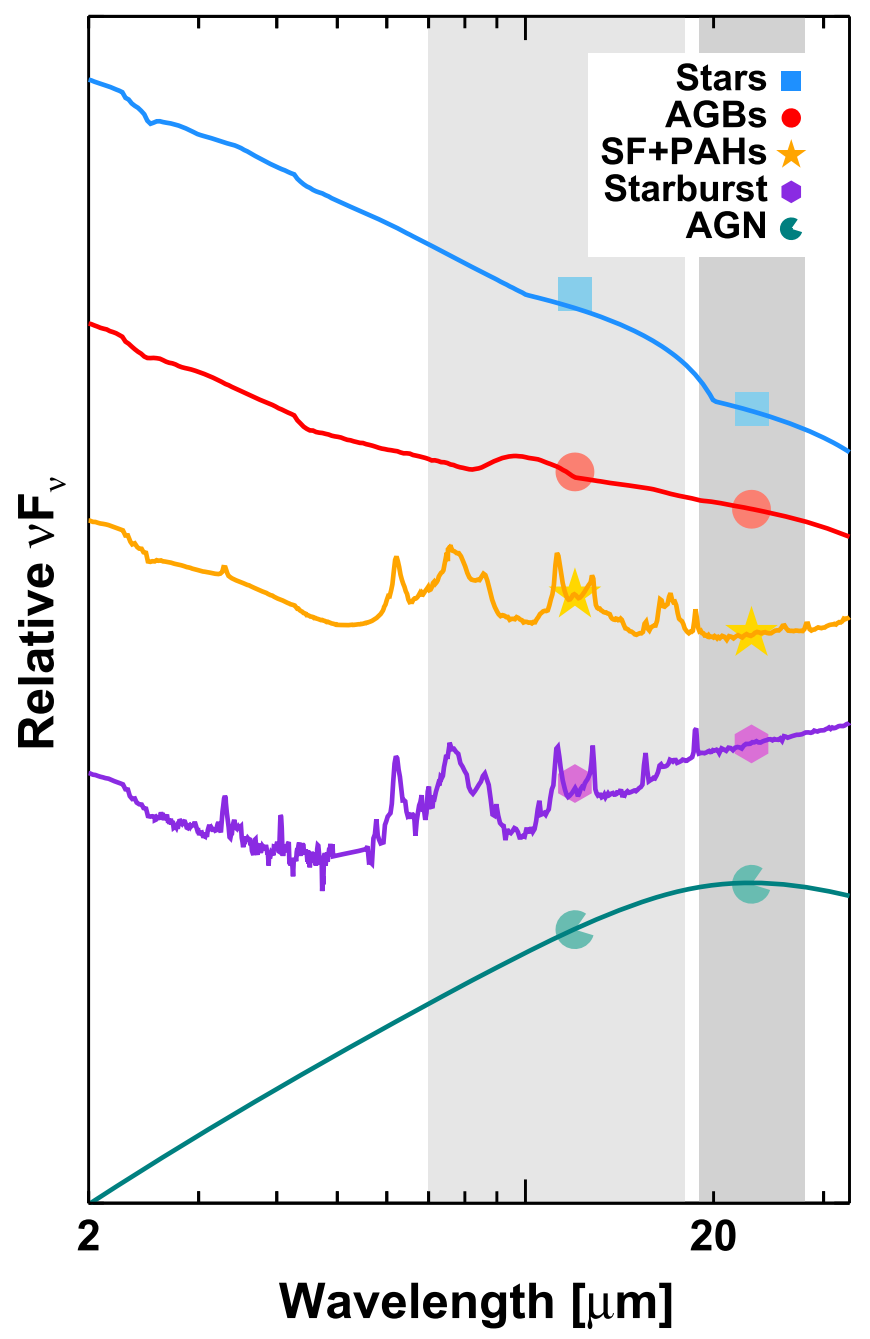

Figure 9. 2-30 $\mu \mathrm{m}$ region of various models of different objects, including a pure stellar population (blue; Bruzual \& Charlot 2003), AGB stars (red; Piovan et al. 2003), and star-forming galaxies including PAH emission (orange; Brown et al. 2014), starbursts (purple; Brown et al. 2014), and an AGN (teal; Sajina et al. 2012). The grayed regions represent the bandwidth of the WISE $12 \mu \mathrm{m}$ and $22 \mu \mathrm{m}$ filters. The blocks show the mean value of each model across the WISE bands. In all cases except for that of the starburst and AGN, it appears that the SEDs decrease with increasing wavelength. 
regions from prolific star formation. This leaves open the possibility that the remnant hot dust emission seen is being heated by low-luminosity AGNs.

Assuming that $100 \%$ of the derived hot dust luminosity in the post-starburst galaxies originates from an AGN, we estimated the Eddington ratios for each 2MASS XSC post-starburst, which is detailed in Appendix $\mathrm{C}$. The resultant Eddington ratios range between $10^{-2}-10^{-4}$, with a peak around $10^{-3}$. This firmly places these possible post-starburst AGNs into the low-luminosity regime. These Eddington ratios are also strict upper limits, as it is unlikely that in all sources, $100 \%$ of the hot dust luminosity is due to an AGN (since intermediate-aged stars also produce hot dust emission in post-starbursts as well).

To determine the X-ray properties of the 2MASS XSC poststarbursts, we cross-matched them with the Swift 70 month BAT All-sky Hard X-ray Survey (Baumgartner et al. 2013) but were unable to match any objects. Given the low Eddington ratio upper limits that the post-starburst 2MASS XSC samples, this is unsurprising, (the flux limit of the Swift BAT catalog is $1.03 \times 10^{11} \mathrm{erg} \mathrm{s}^{-1} \mathrm{~cm}^{-2}$, and thus finds mainly highluminosity AGNs).

We also cross-matched the 2MASS XSC post-starbursts with the Chandra archive. Four were targeted by Chandra (ObsIDs 10270-10273; PI Zabludoff), and all four have weak-tomoderate detections above background fluctuations in a $2^{\prime \prime}$ aperture at the center of the galaxy. We use WEBPIMMS to calculate the associated observed $2-10 \mathrm{keV}$ luminosities ranging between $10^{40}$ and $6 \times 10^{40}$, assuming a power law with $\Gamma=1.8$. The corresponding Eddington ratios (assuming $L_{X} \approx 1 / 16 L_{\text {bol }} ;$ Ho 2008) range between $10^{-5}-10^{-4.5} \cdot{ }^{26}$ Another four were serendipitously in the fields of view of other Chandra observations of comparable exposure times but were not detected to significance above the background. That $\mathrm{X}$-rays were detected coincident with the nuclei of many of the post-starbursts that were observed does provide further evidence that post-starbursts may contain an AGN phase, but given the small number statistics that still exist for these objects, is not conclusive.

Our estimate of possible Eddington ratios of the poststarbursts, derived from the hot dust luminosity and combined with the X-ray results, seems to indicate that the presence of these low-luminosity AGNs in post-starbursts is entirely feasible given the estimated energetics. These results also support the conclusion of De Propris \& Melnick (2014) that AGNs in post-starburst galaxies are not radiatively significant. The $\mathrm{H} \alpha$ fluxes necessary to pass the Goto (2007) criterion seem to rule out radiatively significant AGNs, and in the case of the 2MASS XSC post-starbursts, do not likely represent Eddington ratios above $\sim 10^{-3}$. Burying the AGN under a reservoir of optically thick gas significantly impacts the $\mathrm{H} \alpha$ emission that can be observed, while the mid-IR AGN light is able to escape.

The $\mathrm{E}+\mathrm{A}$ criterion selects against the presence of strong AGNs, quasars, and shocked systems (as these objects all emit $\mathrm{H} \alpha$ or [O II] emission). Thus the possibility of AGNs being the source for the mid-IR colors is not a certainty. But new studies have opened up the possibility that there may be buried lowluminosity AGNs in post-starburst galaxies. Recently, significant molecular gas reservoirs have been discovered in post-starburst galaxies (French et al. 2015; Rowlands et al.

\footnotetext{
${ }^{26}$ One of the four sources (that with the highest X-ray flux) did not have sufficiently accurate spectroscopy to measure $\sigma_{\star}$, thus we were unable to derive an accurate Eddington ratio.
}

2015). These CO-rich post-starbursts also exhibit excess $22 \mu \mathrm{m}$ emission compared to their $\mathrm{CO}(1-0)$ emission, diverging from the relation set by the star-forming galaxies (Alatalo et al. 2016b). Additionally, the optical spectra of post-starbursts (despite having weak nebular lines) have LINER-like line emission, consistent with what is observed in low-luminosity AGNs (Yan et al. 2006; Yang et al. 2006). LINER emission also often originates from other sources, such as aged stellar populations (Yan et al. 2006; Sarzi et al. 2010) and shocks (Allen et al. 2008; Rich et al. 2011; Alatalo et al. 2016a), so this LINER emission is also not confirmatory of AGNs. The poststarburst composite SEDs and WISE colors support the possibility that many post-starbursts contain buried AGNs, which is not contradicted by the ionized gas line emission properties, and may even be supported by the presence of gas (and therefore an obscuring column).

A broad statistical analysis on emission line galaxies from SDSS showed that the AGN fraction in disk-dominated starforming galaxies is significantly underestimated (Trump et al. 2015), their signals being overwhelmed by the ionized gas signatures associated with star formation. Bitsakis et al. $(2015,2016)$ note that in compact group galaxies (a known rapidly evolving population), once star formation started shutting down, the fraction of AGN-hosting galaxies increases even as the AGN luminosity decreases. These trends suggest either that weak AGNs were always present in the nuclei of these galaxies but were being outshone by star formation or that weak AGN activity begins during the phase of star formation quenching; it is unclear whether this result is universal. Our results fuel further discussion about whether the quenching of star formation reduces the mid-IR signal that overwhelmed the weak AGN (Trump et al. 2015) or whether AGN fueling is part of the transition process (Hopkins et al. 2008). Studying whether the mid-IR slope changes as a function of the stellar population age in post-starbursts may enable one to discriminate between these two scenarios, but is beyond the scope of this paper.

\subsection{Toward a Comprehensive Selection of Transitioning Galaxies}

Selecting transitioning galaxies has long been a challenging endeavor. Optical colors can be ambiguous (Schawinski et al. 2014); ultraviolet colors tend to be ultra-sensitive to star formation activity down to $1 \%$ mass fractions (Kaviraj et al. 2007a, 2007b; Choi et al. 2009), creating a set of "frosted" early-type galaxy interlopers. Robust spectral classification can be expensive, requiring high signal-to-noise spectra to detect absorption against the stellar continuum, placing the detection and cataloging high-redshift quenched galaxies out of reach. Wild et al. (2014) showed that "super-colors" could be used to identify post-starburst-like galaxies in the redshift range $0.9<z<1.2$, but this method has not been useful at lower redshifts, due to its dependence on the ultraviolet portion of the SED.

Our new work opens yet another door to finding quenching galaxies, using the mid-IR colors. An independent investigation by Ko et al. (2016) found that stacked spectra of mid-IR excess galaxies (defined using [3.4]-[12] WISE colors) showed signs of an intermediate stellar population. Post-starbursts, the bona fide transitioning population, sit in a distinct phase space in the WISE [3.4]-[4.6] versus [4.6]-[12] colors, which, in the era of 
the James Webb Space Telescope, we will be able to observe up to $z \approx 1$.

Our work has also introduced a new challenge to how we identify quenching galaxies. Given that some post-starburst sources show signs of the presence of an AGN, it is likely that we are missing a significant population of quenching galaxies simply because we are removing all galaxies with significant emission in either $\mathrm{H} \alpha$ or [O II], which an AGN will excite. That AGNs are present in $\mathrm{E}+\mathrm{A}$ galaxies whose selection criteria directly select against them tells us that the AGNs are a crucial ingredient to study when trying to understand the nature of quenching galaxies, and should not be excluded when attempting to create a complete picture of galaxy metamorphosis, even at $z=0$ (and especially at high redshift).

\section{Summary}

We have analyzed the mid-IR properties of a selection of post-starburst galaxies selected through the "E+A" criterion by Goto (2007) from SDSS DR7 (Abazajian et al. 2009). Of the original 564 post-starbursts, we were able to analyze the colors of 534 objects with robust detections from both SDSS and WISE 3.4, 4.6, and $12 \mu \mathrm{m}$. We further investigated poststarbursts detected in the 2MASS XSC, totaling 190 objects, of which 158 have robust $3.4 \mu \mathrm{m}, 4.6 \mu \mathrm{m}$ and $12 \mu \mathrm{m}$ detections. 53 of the 2MASS XSC post-starbursts are robustly $(\mathrm{S} / \mathrm{N}>3)$ detected in the WISE $22 \mu \mathrm{m}$ band. Using these samples, we came to the following conclusions.

The 534 post-starburst galaxies studied have transitioning $u-r$ and [4.6]-[12] colors, falling within the IRTZ discussed by Alatalo et al. (2014a).

After correcting for redshift effects, the [3.4]-[4.6] versus [4.6]-[12] colors of post-starburst galaxies stand out from the colors of both early-type and late-type galaxies, inhabiting the mid-IR twilight zone. This result shows that galaxies do not transition directly across the mid-IR color gap between the early-type and late-type population, requiring an additional source of mid-IR emission.

The SED of post-starburst galaxies requires the inclusion of either strong neutral $(11.3 \mu \mathrm{m}) \mathrm{PAH}$ emission or a TP-AGB component (with circumstellar dust) to fit the 3-12 $\mu \mathrm{m}$ data. A TP-AGB component would also be consistent with the findings of Yan et al. (2006), which required this component to explain the ionized gas emission seen in post-starbursts.

We used MAGPHYS to fit the SEDs of the XSC poststarbursts, extracting the stellar and hot dust luminosities. We find that post-starbursts have intermediate hot dust luminosities (compared to the stellar), and that the [4.6]-[12] $\mu \mathrm{m} \mathrm{WISE}$ colors are a good proxy for the ratio between $L_{\star}$ and $L_{\text {hot dust }}$.

The composite SEDs of our observed post-starbursts (with $22 \mu \mathrm{m}$ emission detected with $\mathrm{S} / \mathrm{N}>3$ ) suggest that an AGN component is needed to account for the hot dust detected in the $22 \mu \mathrm{m}$ WISE band, but we cannot rule out other possibilities. Stacking the $22 \mu \mathrm{m}$ emission in non-detected post-starbursts was also consistent with the need for a hot dust component to explain a flat mid-IR composite SED. The upper limit to the Eddington ratios inferred from the hot dust luminosity range between $10^{-4}-10^{-2}$, with an average of $10^{-3}$. This suggests that while AGNs might be present, they have low luminosity and are not radiatively dominant in the system.

Identifying galaxies that are transitioning requires a multiwavelength approach, and a closer look at the mid-IR has revealed new and exciting results. WISE colors suggest a path toward photometrically identifying galaxies that are transitioning. SEDs of post-starbursts that include $22 \mu \mathrm{m}$ emission suggest the presence of AGNs may be important for some of them, despite their ionized gas selection biasing against the presence of AGNs. We suggest that neglecting to allow for the presence of AGNs when selecting transitioning galaxies may be presenting a biased picture of how metamorphosis takes place.

K.A. thanks R. Peletier for useful discussions as this manuscript was being prepared, as well as the anonymous referee for excellent suggestions that strengthened the manuscript. Support for K.A. is provided by NASA through Hubble Fellowship grant \#HST-HF2-51352.001 awarded by the Space Telescope Science Institute, which is operated by the Association of Universities for Research in Astronomy, Inc., for NASA, under contract NAS5-26555. P.N.A. is partially supported by funding through Herschel, a European Space Agency Cornerstone Mission with significant participation by NASA, through an award issued by JPL/Caltech. T.B. would like to acknowledge support from the CONACyT Research Fellowships program. S.L.C. was supported by ALMACONICYT program 31110020. J.F.B. acknowledges support from grant AYA2016-77237-C3-1-P from the Spanish Ministry of Economy and Competitiveness (MINECO). L.L. acknowledges support for this work provided by NASA through an award issued by JPL/Caltech. K.N. acknowledges support from NASA through the Spitzer Space Telescope. L.J.K. and A.M.M. acknowledge the support of the Australian Research Council (ARC) through Discovery project DP130103925. L.C. received funding from the European Union Seventh Framework Programme (FP7/2007-2013) under grant agreement $n$ 312725. Support for A.M.M. is provided by NASA through Hubble Fellowship grant \#HST-HF2-51377 awarded by the Space Telescope Science Institute, which is operated by the Association of Universities for Research in Astronomy, Inc., for NASA, under contract NAS5-26555.

This publication makes use of data products from the Widefield Infrared Survey Explorer, which is a joint project of the University of California, Los Angeles, and the Jet Propulsion Laboratory/California Institute of Technology, funded by the National Aeronautics and Space Administration. The National Radio Astronomy Observatory is a facility of the National Science Foundation operated under cooperative agreement by Associated Universities, Inc.

Facilities: Sloan, WISE.

\section{Appendix A Fitting the SEDs}

We performed UV-to-mid-IR SED fitting to estimate the physical properties of the galaxies both in our sample and the comparison samples. The code we used is called MAGPHYS and it is described analytically in da Cunha et al. (2008). It is based on the global energy balance between the energy absorbed in the UV and re-emitted in the IR, and adopts a Bayesian approach that draws from a large library of random models encompassing many parameter combinations, such as the star formation histories, metallicities, and dust properties. The theoretical stellar models are computed by the Bruzual \& Charlot (2003) population synthesis code, using the initial mass function presented in Chabrier (2003), whereas the dust models are from Charlot \& Fall (2000). The code compares the 

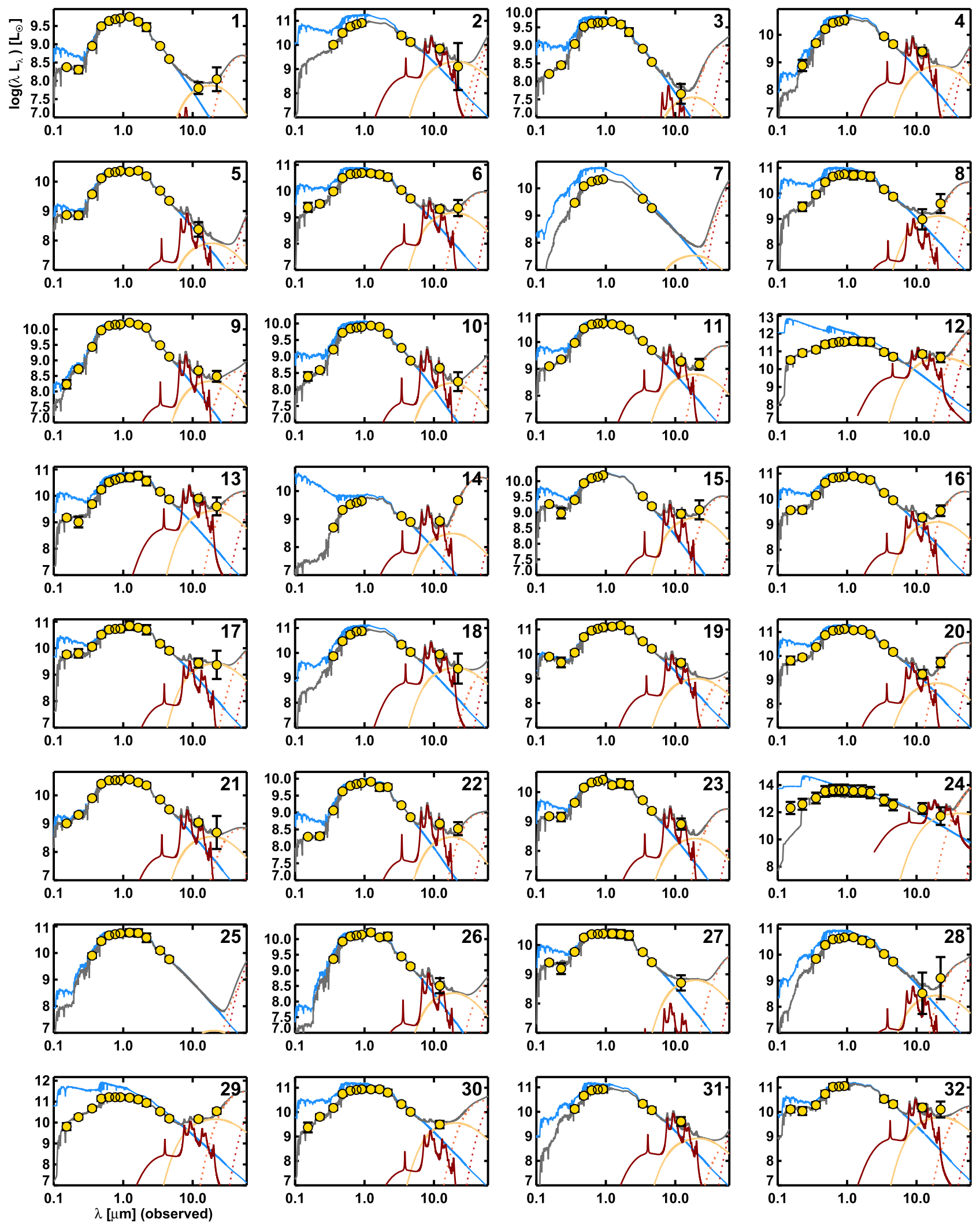

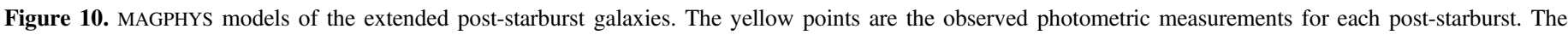

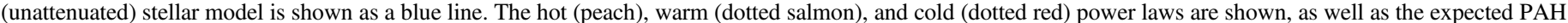
(maroon) emission. The complete fitted model is the dark gray line. (An extended version of this figure is available.)

theoretical models with the observed SED of each galaxy and computes the $\chi^{2}$ value in order to build a probability distribution function (PDF) of each parameter. The final value of each parameter is thus the mean value of the PDF and the uncertainty associated is that given by the 16th and 84th percentiles of the distribution. 
MAGPHYS fits multiple components to the re-emitted IR of each galaxy's SED, including PAH emission, two mid-IR dust components: hot $\left(T_{\text {dust }}=130\right.$ or $250 \mathrm{~K}$ components $)$ and one warm $\left(T_{\text {dust }}=30-60 \mathrm{~K}\right)$ component, and finally a cold dust component. Figure 10 shows the SEDs and MAGPHYS fits to all 2MASS XSC post-starbursts. In the majority of cases, the MAGPHYS fits are good, and are better in cases where both 12 and $22 \mu \mathrm{m}$ emission is detected. The stellar (blue), PAH (maroon), and hot (peach) dust luminosities combine for the composite model (gray), which is shown in the panels in Figure 10.

\section{Appendix B \\ Comparison Sample Selection}

The comparison samples for the spirals and early-type galaxies used originate from the Galaxy Zoo project (Lintott et al. 2008, 2011). The AGN sub-selection within Galaxy Zoo originates from Schawinski et al. (2010). The objects directly modeled were selected randomly from the corresponding catalogs. All objects are required to have available SDSS spectroscopic redshift and photometry in SDSS Data Release 9 (Abazajian et al. 2009).

The early-type galaxy and spiral samples were chosen from the Galaxy Zoo Data Release 2 catalog (Willett et al. 2013), with corresponding morphological classifications (not being classified as AGN-hosting). They contain 232 and 194 galaxies, respectively.

The AGN sample was chosen from the AGN Host Galaxies catalog (Schawinski et al. 2010) and comprises 392 AGNhosting galaxies. This catalog contains a volume-limited sample $\left(0.02<z<0.05, M_{z}<-19.5 \mathrm{AB}\right)$ with emission line classifications consistent with those of AGN-hosting galaxies.

The UV data were obtained from the Galaxy Evolution Explorer All Sky Survey Data Release 6 (Morrissey et al. 2007), resulting in far-ultraviolet (FUV; $1540 \AA$ ) and near-ultraviolet (NUV; $2300 \AA$ ) measurements. All photometric measurements were automatically performed using SEXTRACTOR (see Bertin \& Arnouts 1996). Finally, we used the WISE mid-IR photometry from Lang et al. (2016). These authors performed the "forced photometry" technique in a consistent set of sources between SDSS and WISE, taking advantage of the high resolution of SDSS images to interpret the WISE data.

\section{Appendix C \\ Calculating the Possible Eddington Ratios from the Hot Dust Luminosities}

To determine the possible Eddington ratios of the poststarbursts, we derived values for black hole masses and potential AGN luminosities. We first obtained estimates of the stellar velocity dispersions from SDSS DR9 (Ahn et al. 2012) for the 190 2MASS XSC post-starbursts. We then estimated black hole masses using the $M .-\sigma$ relation from formula 7 in Kormendy \& Ho (2013). The Eddington luminosity was then derived using $L_{\text {Edd }} / L_{\odot}=3.2 \times 10^{4} M_{\bullet} / M_{\odot}$. The possible bolometric luminosities represented by these post-starbursts were estimated by assuming that $100 \%$ of the MAGPHYSderived hot dust luminosity originated from an AGN (Sajina et al. 2005; Richards et al. 2006; Lusso et al. 2013); thus we can estimate the bolometric luminosity of the AGN to be approximately $5 \times L_{\text {hot }}$.

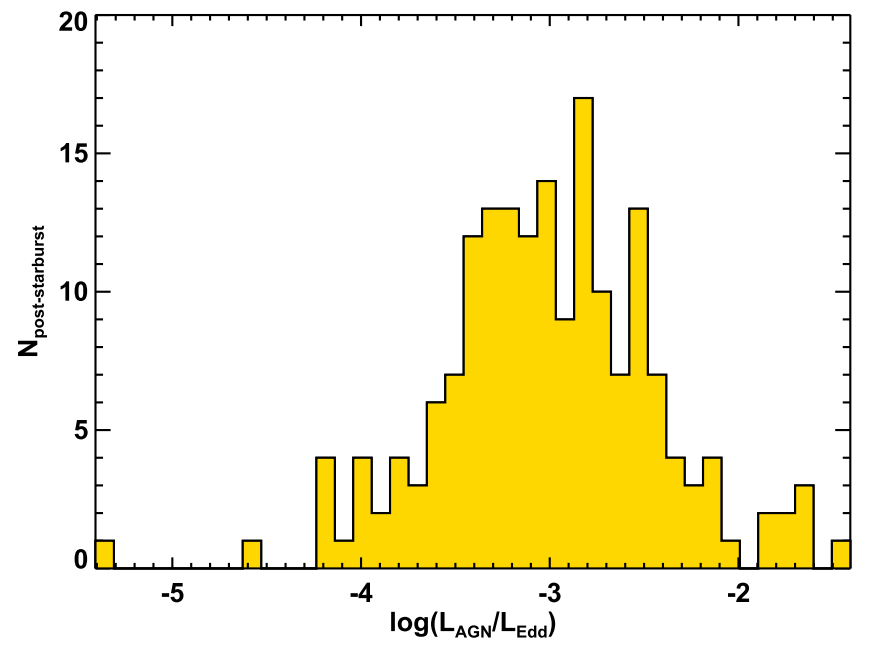

Figure 11. The estimated upper limits to the Eddington ratios represented by the mid-IR hot dust luminosities of the post-starbursts. The estimated ratios firmly place the potential AGNs in post-starbursts into the low-luminosity AGN regime.

Figure 11 shows the Eddington ratio distribution for the post-starbursts, assuming that the entire hot dust luminosity originates from an AGN. The derived Eddington ratios range between $10^{-4}$ and $10^{-2}$, with a peak around $10^{-3}$. This firmly places the post-starbursts into the low-luminosity regime, consistent with the findings of Brown et al. (2009) and De Propris \& Melnick (2014), based on X-ray measurements.

\section{References}

Aalto, S., Costagliola, F., Muller, S., et al. 2016, A\&A, 590, A73 Aalto, S., Muller, S., Sakamoto, K., et al. 2012, A\&A, 546, A68 Abazajian, K. N., Adelman-McCarthy, J. K., the SDSS Collaboration, et al. 2009, ApJS, 182, 543

Abramson, L. E., Kelson, D. D., Dressler, A., et al. 2014, ApJL, 785, L36

Adelman-McCarthy, J. K., Agüeros, M. A., Allam, S. S., et al. 2007, ApJS, 172,634

Ahn, C. P., Alexandroff, R., the SDSS Collaboration, et al. 2012, ApJS, 203, 21

Alatalo, K. 2015, ApJL, 801, L17

Alatalo, K., Appleton, P. N., Lisenfeld, U., et al. 2015a, ApJ, 812, 117

Alatalo, K., Blitz, L., Young, L. M., et al. 2011, ApJ, 735, 88

Alatalo, K., Cales, S. L., Appleton, P. N., et al. 2014a, ApJL, 794, L13

Alatalo, K., Cales, S. L., Rich, J. A., et al. 2016a, ApJS, 224, 38

Alatalo, K., Lacy, M., Lanz, L., et al. 2015b, ApJ, 798, 31

Alatalo, K., Lisenfeld, U., Lanz, L., et al. 2016b, ApJ, 827, 106

Alatalo, K., Nyland, K., Graves, G., et al. 2014b, ApJ, 780, 186

Allen, M. G., Groves, B. A., Dopita, M. A., et al. 2008, ApJS, 178, 20

Appleton, P. N., Guillard, P., Boulanger, F., et al. 2013, ApJ, 777, 66

Assef, R. J., Stern, D., Kochanek, C. S., et al. 2013, ApJ, 772, 26

Baade, W. 1958, RA, 5, 3

Baldry, I. K., Glazebrook, K., Brinkmann, J., et al. 2004, ApJ, 600, 681

Baumgartner, W. H., Tueller, J., Markwardt, C. B., et al. 2013, ApJS, 207, 19

Becker, R. H., White, R. L., \& Helfand, D. J. 1995, ApJ, 450, 559

Beirão, P., Brandl, B. R., Appleton, P. N., et al. 2008, ApJ, 676, 304

Bekki, K. 1998, ApJL, 502, L133

Bekki, K., Couch, W. J., \& Shioya, Y. 2002, ApJ, 577, 651

Bertin, E., \& Arnouts, S. 1996, A\&AS, 117, 393

Bitsakis, T., Charmandaris, V., Appleton, P. N., et al. 2014, A\&A, 565, A25 Bitsakis, T., Charmandaris, V., da Cunha, E., et al. 2011, A\&A, 533, A142

Bitsakis, T., Dultzin, D., Ciesla, L., et al. 2015, MNRAS, 450, 3114

Bitsakis, T., Dultzin, D., Ciesla, L., et al. 2016, MNRAS, 459, 957

Blanton, M. R., \& Moustakas, J. 2009, ARA\&A, 47, 159

Borthakur, S., Yun, M. S., \& Verdes-Montenegro, L. 2010, ApJ, 710, 385

Boselli, A., Ciesla, L., Buat, V., et al. 2010, A\&A, 518, L61

Bregman, J. D., Bregman, J. N., \& Temi, P. 2008, in ASP Conf. Ser. 381, Infrared Diagnostics of Galaxy Evolution, ed. R.-R. Chary, H. I. Teplitz, \& K. Sheth (San Francisco, CA: ASP), 34 
Bressan, A., Panuzzo, P., Buson, L., et al. 2006, ApJL, 639, L55 Brown, M. J. I., Jarrett, T. H., \& Cluver, M. E. 2014, ApJS, 212, 18 Brown, M. J. I., Moustakas, J., Caldwell, N., et al. 2009, ApJ, 703, 150 Bruzual, G., \& Charlot, S. 2003, MNRAS, 344, 1000 Bryant, P. M., \& Scoville, N. Z. 1999, AJ, 117, 2632 Cales, S. L., \& Brotherton, M. S. 2015, MNRAS, 449, 2374

Cales, S. L., Brotherton, M. S., Shang, Z., et al. 2011, ApJ, 741, 106 Cales, S. L., Brotherton, M. S., Shang, Z., et al. 2013, ApJ, 762, 90 Calzetti, D., Kennicutt, R. C., Engelbracht, C. W., et al. 2007, ApJ, 666, 870 Canalizo, G., \& Stockton, A. 2000, AJ, 120, 1750 Canalizo, G., \& Stockton, A. 2001, ApJ, 555, 719 Canalizo, G., \& Stockton, A. 2013, ApJ, 772, 132 Chabrier, G. 2003, PASP, 115, 763

Charlot, S., \& Fall, S. M. 2000, ApJ, 539, 718

Chilingarian, I. V., \& Zolotukhin, I. Y. 2012, MNRAS, 419, 1727

Chisari, N. E., \& Kelson, D. D. 2012, ApJ, 753, 94

Choi, Y., Goto, T., \& Yoon, S.-J. 2009, MNRAS, 395, 637

Cicone, C., Feruglio, C., Maiolino, R., et al. 2012, A\&A, 543, A99

Cicone, C., Maiolino, R., Sturm, E., et al. 2014, A\&A, 562, A21

Ciesla, L., Boquien, M., Boselli, A., et al. 2014, A\&A, 565, A128

Costagliola, F., Herrero-Illana, R., Lohfink, A., et al. 2016, A\&A, 594, A114 Croton, D. J., Springel, V., White, S. D. M., et al. 2006, MNRAS, 365, 11

da Cunha, E., Charlot, S., \& Elbaz, D. 2008, MNRAS, 388, 1595

Davis, T. A., Krajnović, D., McDermid, R. M., et al. 2012, MNRAS, 426, 1574

Davis, T. A., Young, L. M., Crocker, A. F., et al. 2014, MNRAS, 444, 3427 De Propris, R., \& Melnick, J. 2014, MNRAS, 439, 2837

Di Matteo., T., Springel, V., \& Hernquist, L. 2005, Natur, 433, 604

Dressler, A., \& Gunn, J. E. 1983, ApJ, 270, 7

Dressler, A., Oemler, A., Jr, Poggianti, B. M., et al. 2013, ApJ, 770, 62

Eliche-Moral, M. C., González-García, A. C., Aguerri, J. A. L., et al. 2012, A\&A, 547, A48

Feruglio, C., Maiolino, R., Piconcelli, E., et al. 2010, A\&A, 518, L155

Fischer, J., Sturm, E., González-Alfonso, E., et al. 2010, A\&A, 518, L41

French, K. D., Yang, Y., Zabludoff, A., et al. 2015, ApJ, 801, 1

Goto, T. 2005, MNRAS, 357, 937

Goto, T. 2007, MNRAS, 377, 1222

Guillard, P., Boulanger, F., Lehnert, M. D., et al. 2015, A\&A, 574, A32

Hayward, C. C., Lanz, L., Ashby, M. L. N., et al. 2014, MNRAS, 445, 1598

Hickson, P., Mendes de Oliveira, C., Huchra, J. P., \& Palumbo, G. G. 1992, ApJ, 399, 353

Ho, L. C. 2008, ARA\&A, 46, 475

Holmberg, E. 1958, MeLuS, 136, 1

Hopkins, P. F., Hernquist, L., Cox, T. J., et al. 2006, ApJS, 163, 1

Hopkins, P. F., Hernquist, L., Cox, T. J., \& Kereš, D. 2008, ApJS, 175, 356

Hubble, E. P. 1926, ApJ, 64, 321

Johnson, K. E., Hibbard, J. E., Gallagher, S. C., et al. 2007, AJ, 134, 1522

Kaneda, H., Onaka, T., \& Sakon, I. 2005, ApJL, 632, L83

Kaneda, H., Onaka, T., Sakon, I., et al. 2008, ApJ, 684, 270

Kannappan, S. J., Stark, D. V., Eckert, K. D., et al. 2013, ApJ, 777, 42

Kaviraj, S., Kirkby, L. A., Silk, J., \& Sarzi, M. 2007a, MNRAS, 382, 960

Kaviraj, S., Schawinski, K., Devriendt, J. E. G., et al. 2007b, ApJS, 173, 619

Kaviraj, S., Shabala, S. S., Deller, A. T., \& Middelberg, E. 2015, MNRAS, 452, 774

Kelson, D. D., \& Holden, B. P. 2010, ApJL, 713, L28

Ko, J., Chung, H., Hwang, H. S., \& Lee, J. C. 2016, ApJ, 820, 132

Ko, J., Hwang, H. S., Lee, J. C., \& Sohn, Y.-J. 2013, ApJ, 767, 90

Kormendy, J., \& Ho, L. C. 2013, ARA\&A, 51, 511

Kriek, M., Labbé, I., Conroy, C., et al. 2010, ApJL, 722, L64

Lang, D., Hogg, D. W., \& Schlegel, D. J. 2016, AJ, 151, 36

Lanz, L., Ogle, P. M., Alatalo, K., \& Appleton, P. N. 2016, ApJ, 826, 29

Lanz, L., Ogle, P. M., Evans, D., et al. 2015, ApJ, 801, 17

Lintott, C., Schawinski, K., Bamford, S., et al. 2011, MNRAS, 410, 166

Lintott, C. J., Schawinski, K., Slosar, A., et al. 2008, MNRAS, 389, 1179

Lisenfeld, U., Appleton, P. N., Cluver, M. E., et al. 2014, A\&A, 570, A24

Lusso, E., Hennawi, J. F., Comastri, A., et al. 2013, ApJ, 777, 86

Malmquist, K. G. 1925, MeLuF, 106, 1

Maraston, C. 2005, MNRAS, 362, 799

Maraston, C., Pforr, J., Henriques, B. M., et al. 2013, MNRAS, 435, 2764

Martig, M., Bournaud, F., Teyssier, R., \& Dekel, A. 2009, ApJ, 707, 250

Martig, M., Crocker, A. F., Bournaud, F., et al. 2013, MNRAS, 432, 1914

Martinez-Badenes, V., Lisenfeld, U., Espada, D., et al. 2012, A\&A, 540, A96

Mateos, S., Carrera, F. J., Alonso-Herrero, A., et al. 2015, MNRAS, 449, 1422
Matsuoka, Y., Strauss, M. A., Shen, Y., et al. 2015, ApJ, 811, 91

Melnick, J., \& De Propris, R. 2013, MNRAS, 431, 2034

Meusinger, H., Brünecke, J., Schalldach, P., \& in der Au, A. 2017, A\&A, 597, A134

Mihos, J. C. 1995, ApJL, 438, L75

Mihos, J. C., \& Hernquist, L. 1996, ApJ, 464, 641

Moore, B., Katz, N., Lake, G., Dressler, A., \& Oemler, A. 1996, Natur, 379,613

Morrissey, P., Conrow, T., Barlow, T. A., et al. 2007, ApJS, 173, 682

Nielsen, D. M., Ridgway, S. E., De Propris, R., \& Goto, T. 2012, ApJL, 761, L16

Oppenheimer, B. D., Davé, R., Kereš, D., et al. 2010, MNRAS, 406, 2325

Panuzzo, P., Vega, O., Bressan, A., et al. 2007, ApJ, 656, 206

Peletier, R. F., Kutdemir, E., van der Wolk, G., et al. 2012, MNRAS, 419, 2031

Piovan, L., Tantalo, R., \& Chiosi, C. 2003, A\&A, 408, 559

Qu, Y., Di Matteo, P., Lehnert, M., van Driel, W., \& Jog, C. J. 2010, A\&A, 515, A11

Quintero, A. D., Hogg, D. W., Blanton, M. R., et al. 2004, ApJ, 602, 190

Rasmussen, J., Ponman, T. J., Verdes-Montenegro, L., Yun, M. S., \& Borthakur, S. 2008, MNRAS, 388, 1245

Rich, J. A., Kewley, L. J., \& Dopita, M. A. 2011, ApJ, 734, 87

Richards, G. T., Lacy, M., Storrie-Lombardi, L. J., et al. 2006, ApJS, 166, 470

Roseboom, I. G., Oliver, S., \& Farrah, D. 2009, ApJL, 699, L1

Rowlands, K., Wild, V., Nesvadba, N., et al. 2015, MNRAS, 448, 258

Sajina, A., Lacy, M., \& Scott, D. 2005, ApJ, 621, 256

Sajina, A., Yan, L., Fadda, D., Dasyra, K., \& Huynh, M. 2012, ApJ, 757, 13

Sarzi, M., Shields, J. C., Schawinski, K., et al. 2010, MNRAS, 402, 2187

Schawinski, K., Thomas, D., Sarzi, M., et al. 2007, MNRAS, 382, 1415

Schawinski, K., Urry, C. M., Simmons, B. D., et al. 2014, MNRAS, 440, 889

Schawinski, K., Urry, C. M., Virani, S., et al. 2010, ApJ, 711, 284

Shin, M.-S., Strauss, M. A., \& Tojeiro, R. 2011, MNRAS, 410, 1583

Silk, J., \& Rees, M. J. 1998, A\&A, 331, L1

Silva, L., Granato, G. L., Bressan, A., \& Danese, L. 1998, ApJ, 509, 103

Skrutskie, M. F., Cutri, R. M., Stiening, R., et al. 2006, AJ, 131, 1163

Smith, J. D. T., Draine, B. T., Dale, D. A., et al. 2007, ApJ, 656, 770

Spergel, D. N., Bean, R., Doré, O., et al. 2007, ApJS, 170, 377

Springel, V., Di Matteo, T., \& Hernquist, L. 2005, ApJL, 620, L79

Strateva, I., Ivezić, Ž, Knapp, G. R., et al. 2001, AJ, 122, 1861

Sturm, E., González-Alfonso, E., Veilleux, S., et al. 2011, ApJL, 733, L16

Sturm, E., Lutz, D., Tran, D., et al. 2000, A\&A, 358, 481

Suh, K.-W., \& Kwon, Y.-J. 2011, MNRAS, 417, 3047

Taylor, M. B. 2005, in ASP Conf. Ser. 347, Astronomical Data Analysis Software and Systems XIV, ed. P. Shopbell, M. Britton, \& R. Ebert (San Francisco, CA: ASP), 29

Temi, P., Brighenti, F., \& Mathews, W. G. 2007, ApJ, 660, 1215

Tinsley, B. M. 1978, ApJ, 222, 14

Toomre, A., \& Toomre, J. 1972, ApJ, 178, 623

Trump, J. R., Sun, M., Zeimann, G. R., et al. 2015, ApJ, 811, 26

Utomo, D., Kriek, M., Labbé, I., Conroy, C., \& Fumagalli, M. 2014, ApJL, 783, L30

van der Wolk, G. 2011, PhD thesis, Groningen

Vazdekis, A., Sánchez-Blázquez, P., Falcón-Barroso, J., et al. 2010, MNRAS, 404, 1639

Vega, O., Bressan, A., Panuzzo, P., et al. 2010, ApJ, 721, 1090

Verdes-Montenegro, L., Yun, M. S., Williams, B. A., et al. 2001, A\&A, 377,812

Wild, V., Almaini, O., Cirasuolo, M., et al. 2014, MNRAS, 440, 1880

Willett, K. W., Lintott, C. J., Bamford, S. P., et al. 2013, MNRAS, 435 2835

Wright, E. L., Eisenhardt, P. R. M., Mainzer, A. K., et al. 2010, AJ, 140, 1868

Xilouris, E. M., Madden, S. C., Galliano, F., Vigroux, L., \& Sauvage, M. 2004, A\&A, 416, 41

Yan, R., Newman, J. A., Faber, S. M., et al. 2006, ApJ, 648, 281

Yang, Y., Tremonti, C. A., Zabludoff, A. I., \& Zaritsky, D. 2006, ApJL, 646, L33

Yesuf, H. M., Faber, S. M., Trump, J. R., et al. 2014, ApJ, 792, 84

Young, L. M., Scott, N., Serra, P., et al. 2014, MNRAS, 444, 3408

Zabludoff, A. I., \& Mulchaey, J. S. 1998, ApJ, 496, 39

Zabludoff, A. I., Zaritsky, D., Lin, H., et al. 1996, ApJ, 466, 104

Zakamska, N. L., Hamann, F., Pâris, I., et al. 2016, MNRAS, 459, 3144

Zibetti, S., Gallazzi, A., Charlot, S., Pierini, D., \& Pasquali, A. 2013, MNRAS, 428, 1479 